San Jose State University

SJSU ScholarWorks

Master's Theses

Master's Theses and Graduate Research

Summer 2019

\title{
Signal Integrity Optimization of RF/Microwave Transmission Lines in Multilayer PCBs
}

Justin Thien Le

San Jose State University

Follow this and additional works at: https://scholarworks.sjsu.edu/etd_theses

\section{Recommended Citation}

Le, Justin Thien, "Signal Integrity Optimization of RF/Microwave Transmission Lines in Multilayer PCBs" (2019). Master's Theses. 5036.

DOI: https://doi.org/10.31979/etd.4ddq-zyef

https://scholarworks.sjsu.edu/etd_theses/5036

This Thesis is brought to you for free and open access by the Master's Theses and Graduate Research at SJSU ScholarWorks. It has been accepted for inclusion in Master's Theses by an authorized administrator of SJSU ScholarWorks. For more information, please contact scholarworks@sjsu.edu. 


\title{
SIGNAL INTEGRITY OPTIMIZATION OF RF/MICROWAVE TRANSMISSION LINES IN MULTILAYER PCBS
}

\author{
A Thesis \\ Presented to \\ The Faculty of the Department of Electrical Engineering \\ San José State University \\ In Partial Fulfillment \\ of the Requirements for the Degree \\ Master of Science
}

by

Justin T. Le

August 2019 
(C) 2019

Justin T. Le

ALL RIGHTS RESERVED 
The Designated Thesis Committee Approves the Thesis Titled

\title{
SIGNAL INTEGRITY OPTIMIZATION OF RF/MICROWAVE TRANSMISSION LINES IN MULTILAYER PCBS
}

\author{
by \\ Justin T. Le \\ APPROVED FOR THE DEPARTMENT OF ELECTRICAL ENGINEERING \\ SAN JOSÉ STATE UNIVERSITY
}

August 2019

Lili He, Ph.D.

Raymond S. Kwok, Ph.D.

Hiu Yung Wong, Ph.D.
Department of Electrical Engineering

Department of Electrical Engineering

Department of Electrical Engineering 


\title{
ABSTRACT \\ SIGNAL INTEGRITY OPTIMIZATION OF RF/MICROWAVE TRANSMISSION LINES IN MULTILAYER PCBS
}

\author{
by Justin T. Le
}

While allowing for flexible trace routing and device miniaturization, multilayer printed circuit boards (PCB) suffer from performance issues at high frequency due to the impedance mismatch caused by vertical transitions. In this paper, a process for optimizing the high-speed performance of microstrip to stripline transitions in multilayer PCBs is demonstrated. This includes strategic tuning of via dimensions using timedomain reflectometry and an analysis of the use of shielding vias to prevent parasitic cavity resonance. Simulations of optimized 2-layer, 4-layer, and 6-layer microstrip to stripline transitions show a return loss of $20 \mathrm{~dB}$ up to $7 \mathrm{GHz}$. To demonstrate a useful microwave application, a planar filter with a passband of $4 \mathrm{GHz}$ to $6 \mathrm{GHz}$ is submerged 6-layers. Simulation shows that when paired with the optimized vertical transitions, the filter can maintain performance. 


\section{ACKNOWLEDGMENTS}

First, thanks are due to my thesis committee chair, Dr. Lili He, for providing support and for guiding me in my thesis work. In addition, I would like to thank Dr. Ray Kwok for taking me under his wing, offering guidance on my thesis work, and allowing me to be his apprentice at both school and work. I would also like to thank Dr. Hiu Yung Wong for his contributions as a committee member. Furthermore, I would like to thank Vanessa Barahona for bearing with me through my academic pursuit. Lastly, thanks are due to my mom for always supporting me in my life journey. 


\section{TABLE OF CONTENTS}

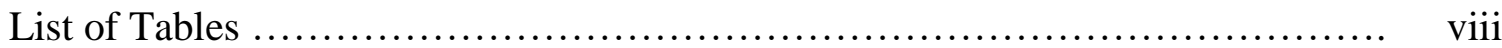

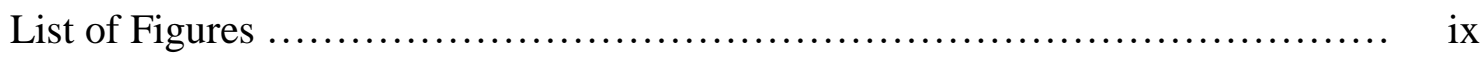

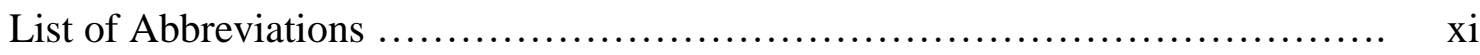

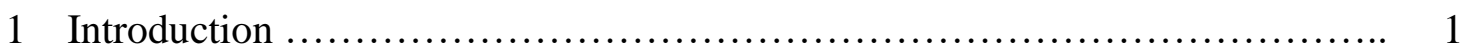

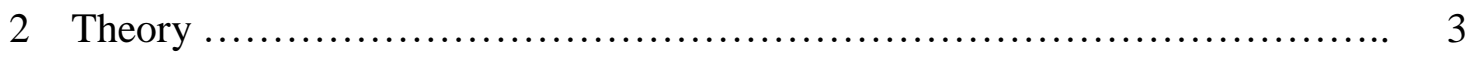

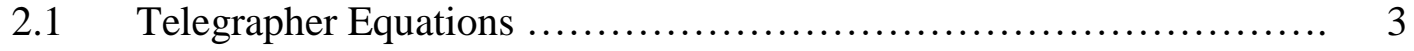

2.1.1 Transmission Lines and Characteristic Impedance .............. 7

2.1.2 Characteristic Impedance of a Coaxial Cable ................... 8

2.2 Impedance Mismatch and Reflection Coefficient .................... 8

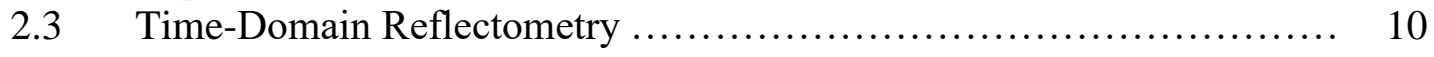

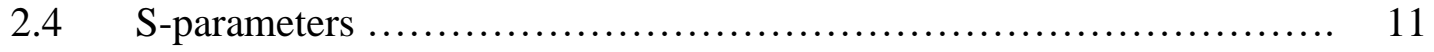

3 Multilayer PCB Manufacturing Process ................................. 13

3.1 General Multilayer Manufacturing Process ......................... 13

3.2 High Frequency Considerations .................................. 14

3.2.1 Material Selection and PTFE ............................. 14

3.2.2 Bonding and Stack-Up ................................ 15

3.2.3 Vertical Connections .................................... 17

4 Literature Review ...................................................... 19

4.1 Lumped Element Model ........................................ 19

4.2 EM Simulation Optimization ..................................... 20

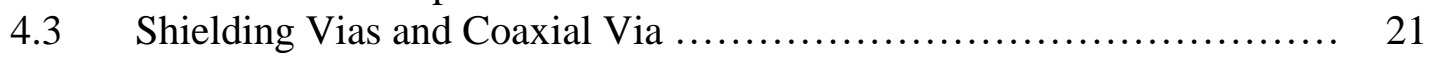

$4.4 \quad$ Nonconventional Vias ....................................... 22

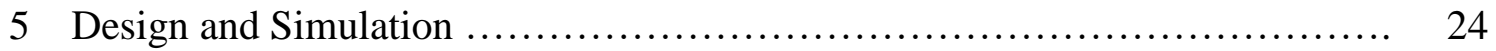

$5.1 \quad$ PCB Stack-Up ................................................ 24

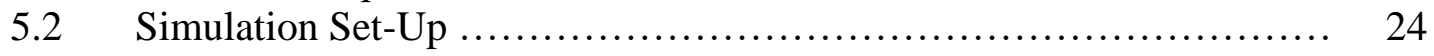

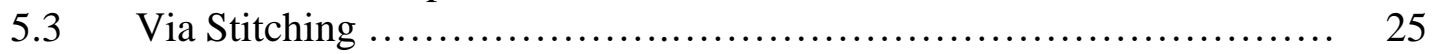

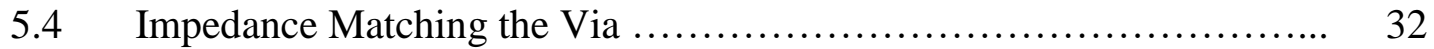

5.4.1 Low Impedance Via .................................... 32

5.4.2 High Impedance Via ................................. 33

5.4.3 Optimized Vias ....................................... 35

$5.5 \quad$ Cascading a Planar Filter ..................................... 37 


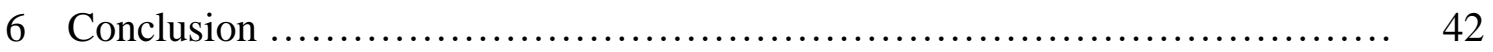

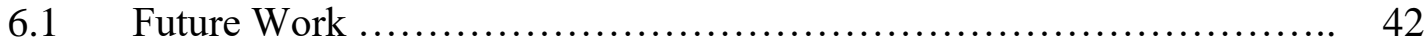

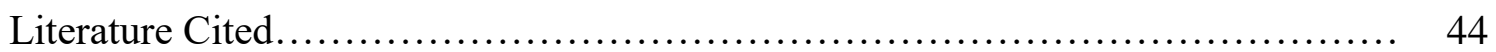




\section{LIST OF TABLES}

Table 1. PTFE Stack-Up Summary ................................... 16

Table 2. Common Bonding Materials .................................. 16

Table 3. Impedance Optimization Guidelines .............................. 20

Table 4. Guidelines for Other Parasitic Effects ............................ 21

Table 5. Via Stitch Spacing ....................................... 30

Table 6. Optimized Via Dimensions and Performance ....................... 36

Table 7. Filter Specifications ....................................... 39 


\section{LIST OF FIGURES}

Fig. 1. Lumped element model of a finite length transmission line ........... 5

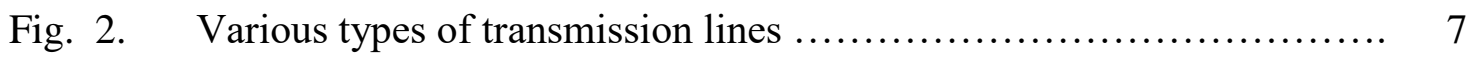

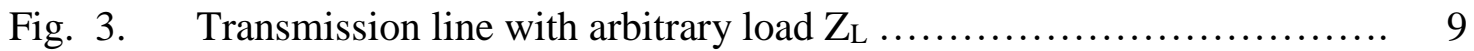

Fig. 4. Bounce diagram of a microstrip with and without a via .............. 11

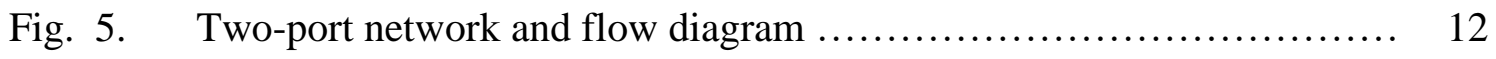

Fig. 6. PTFE stack-up options ........................................ 15

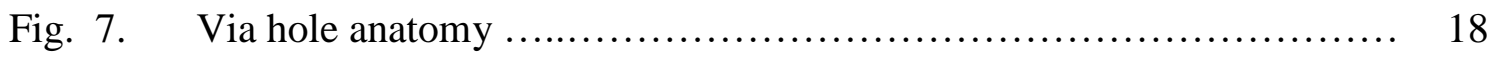

Fig. 8. Lumped element model of a via .................................. 19

Fig. 9. 3D model of 1-level transition with no stitching vias ................. 25

Fig. 10. S-parameters of 1-level transition with no stitching vias .............. 26

Fig. 11. E-field plot of 1-level transition with no stitching vias ............... 26

Fig. 12. 3D Model of 3-level transition with no stitching vias ................ 27

Fig. 13. S-parameters of 3-level transition with no stitching vias ............. 27

Fig. 14. E-field plot of 3-level transition with no stitching vias $\ldots \ldots \ldots \ldots \ldots \ldots .28$

Fig. 15. 3D Model of 2, 4, 6, and 8 stitching vias .......................... 29

Fig. 16. S-parameters of $0,2,4,6$, and 8 stitching vias $\ldots \ldots \ldots \ldots \ldots \ldots \ldots \ldots \ldots \ldots$ 
Fig. 17. E-field plot before and after via stitching ....................... 31

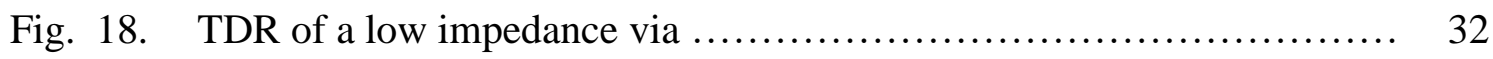

Fig. 19. S-parameters of low impedance via ........................... 33

Fig. 20. TDR of a high impedance via ................................ 34

Fig. 21. S-parameters of high impedance via ........................... 34

Fig. 22. Optimized via for a 1, 2, and 3 level transition $\ldots \ldots \ldots \ldots \ldots \ldots \ldots \ldots \ldots \ldots$

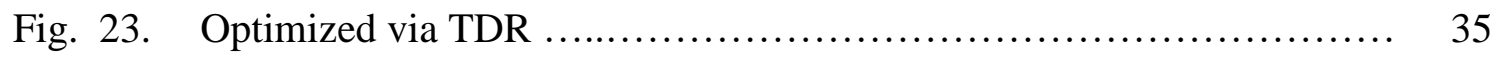

Fig. 24. S-parameters of optimized 1, 2, and 3 level transitions .............. 36

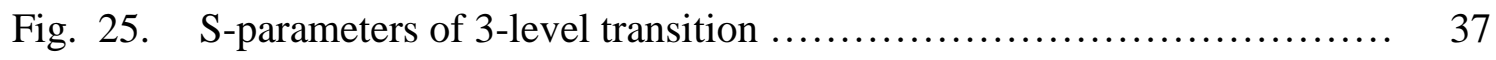

Fig. 26. 3D models of cascaded 1, 2, and 3 level transitions .................... 38

Fig. 27. S-parameters of cascaded 1, 2, and 3 level transitions ................ 38

Fig. 28. 3D model of planar 3-pole filter ............................. 39

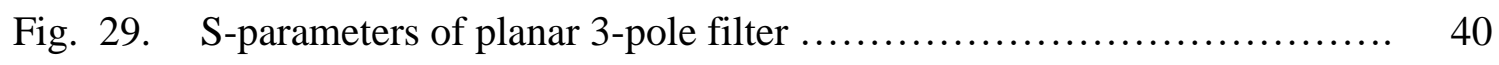

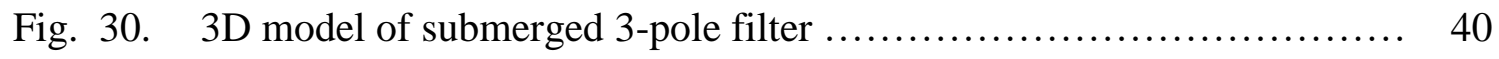

Fig. 31. S-parameters of submerged 3-pole filter ....................... 41

Fig. 32. S-parameters of submerged 3-pole filter after tuning ................ 41 


\section{LIST OF ABBREVIATIONS}

$\begin{array}{ll}\text { CTE } & \text { Coefficient of Thermal Expansion } \\ \text { DC } & \text { Direct Current } \\ \text { EM } & \text { Electromagnetic } \\ \text { EMI } & \text { Electromagnetic Interference } \\ \text { FEP } & \text { Fluorinated Ethylene Propylene } \\ \text { FR-4 } & \text { Fire Resistant Four } \\ \text { LCP } & \text { Liquid Crystalline Polymer } \\ \text { PCB } & \text { Printed Circuit Board } \\ \text { PTFE } & \text { Polytetrafluoroethylene } \\ \text { RF } & \text { Radio Frequency } \\ \text { TDR } & \text { Time-Domain Reflectometry } \\ \text { VIA } & \text { Vertical Interconnect Access } \\ \end{array}$




\section{INTRODUCTION}

With the ever-increasing demand for the miniaturization of electronics, multilayer printed circuit boards (PCB) have been used to realize compact, high density devices. While allowing for size reduction and flexibility in trace routing, the vertical transitions from layer to layer can introduce signal integrity issues at high frequency. This includes impedance mismatch, reflections, electromagnetic interference, bandwidth, mode conversion, and insertion loss. Thus, multilayer PCBs are generally avoided by radio frequency (RF) engineers and high-speed digital designers.

However, the crossover of traces is sometimes necessary and multilayer PCBs can mitigate this issue. For RF designers, a crossover is needed in balanced power amplifiers where the DC bias line is required to cross an RF signal line [1]. One solution has been to use air-bridges or a coaxial cable to cross traces. This is nonideal because it is a nonplanar structure and can increase size, cost, and complexity. In addition, crossovers are also needed in beam-forming networks in which dense transmission line routings are used [2].

In microwave applications, passive planar structures such as filters, couplers, and power dividers can take up significant board space. The ability to bury these passive structures in another layer can allow for a dramatic reduction in board size. Devices such as a filter bank, which consists of multiple filters, must have each filter placed adjacent to one another. The ability to vertically stack each filter could reduce the filter bank size to the size of just one filter. 
The vertical access interconnect, also known as via, is the vertical connection between layers. Vias are most commonly realized as a copper plated, drilled hole and are the primary reason multilayer PCB performance degrades at high frequencies. At low frequencies, these vertical junctions do not present an issue. At high frequencies, any discontinuities in geometry and material properties can cause signal reflections. Because multilayer PCBs are restricted to only planar geometries and copper plated cylindrical holes, it can be difficult to realize a seamless junction in which minimal reflection is achieved. Thus, a planar design technique to optimize the electrical performance of the via is needed.

In effort to mitigate this issue, the work in this thesis uses impedance matching techniques and the concept of characteristic impedance to design an optimal via using electromagnetic simulation. This includes an analysis of several design variations and a general technique for ensuring optimal performance in multilayer PCBs. 


\section{THEORY}

The issue of signal integrity is, in essence, a transmission line theory problem. While it is typically unfamiliar to designers of low frequency circuits, RF engineers have been using transmission line theory as early as World War II. The work in this thesis relies on the core concepts, and it is important that the reader has some background. Thus, this section discusses some of the foundational concepts. This includes the telegrapher equations, characteristic impedance, reflection coefficient, and the S-parameters. In addition, time-domain reflectometry is discussed.

\section{$2.1 \quad$ Telegrapher Equations}

The basis of modern electromagnetic theory was derived by James Clerk Maxwell in a paper published in 1865 titled "A Dynamical Theory of Electromagnetic Field.” From mathematical deduction, Maxwell theorized that light was a form of electromagnetic wave. This theory was then experimentally validated by Hertz. Maxwell's equations are heavily based on the work of Gauss, Ampere, Faraday, and other physicists [3]. Prior to his publication, electric field and magnetic field were considered separate entities, and Maxwell's work unified them to a single entity known as electromagnetic fields. The original Maxwell's equations were mathematically complex and difficult to understand. Oliver Heaviside rewrote the original Maxwell's equations into its modern, well-known vector notation form which are shown below. 


$$
\begin{gathered}
\nabla \times \vec{E}=\frac{-\partial \vec{B}}{\partial t}-\vec{M} \\
\nabla \times \vec{H}=\frac{\partial \vec{D}}{\partial t}+\vec{J} \\
\nabla \cdot \vec{D}=\rho \\
\nabla \cdot \vec{B}=0
\end{gathered}
$$

While Maxwell's equations provide a complete spatial description of electromagnetic fields, they are often impractical to work with for engineering design purposes. Thus, transmission line theory was developed as an extension of circuit theory to account for electromagnetic wave propagation, and it is needed when the wavelength of a signal is proportional to the physical size of the conductor. This is useful since electrical engineers typically work with voltage and current instead of electric field and magnetic field. To account for a propagating voltage or current wave, a finite length transmission line is modeled with the lumped circuit elements shown in Fig. 1. By cascading this equivalent circuit, a transmission line can be modeled using traditional circuit theory. 


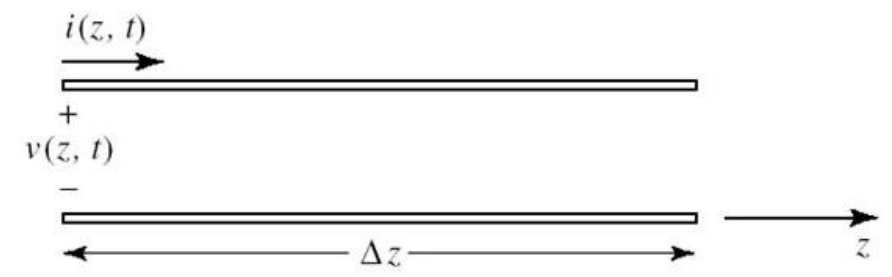

(a)

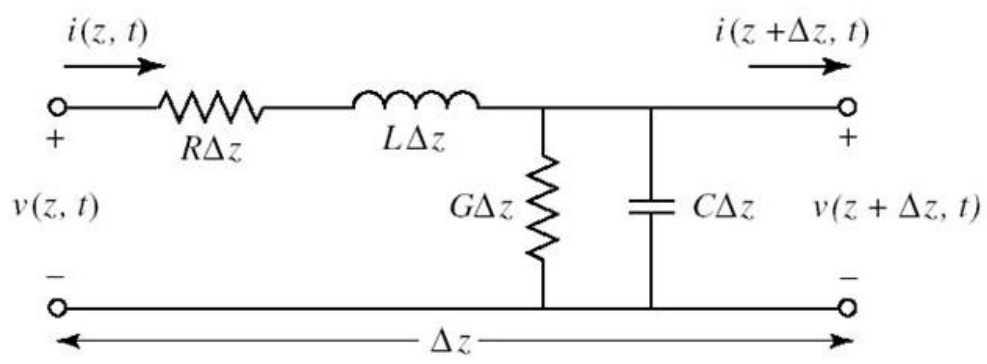

(b)

Fig. 1. Lumped element model of a finite length transmission line [3].

The series inductor represents the self-inductance of the two conductors, and the shunt capacitor represents the capacitance between the two conductors. The series resistor represents the loss due to the finite conductivity of the conductors, and the shunt resistor represents the dielectric loss of the substrate material between the conductors since most of the electromagnetic energy is in the substrate between the signal trace and ground.

This lumped element model yields the telegrapher equations which can be mathematically manipulated to yield useful design parameters such as characteristic impedance. Using Kirkoff's voltage law on the lumped element model yields (5) and Kirkoff's current law yields (6). Rearranging the terms and taking the limit as $\Delta \mathrm{z} \rightarrow 0$, yields (7) and (8). Equations (7) and (8) are known as the telegrapher equations. 


$$
\begin{gathered}
v(z, t)-R \Delta z i(z, t)-L \Delta z \frac{\partial i(z, t)}{\partial t}-v(z+\Delta z, t)=0 \\
i(z, t)-G \Delta z v(z+\Delta z, t)-C \Delta z \frac{\partial v(z+\Delta z, t)}{\partial t}-i(z+\Delta z, t)=0 \\
\frac{\partial v(z, t)}{\partial z}=-R i(z, t)-L \frac{\partial i(z, t)}{\partial t} \\
\frac{\partial i(z, t)}{\partial z}=-G v(z, t)-C \frac{\partial v(z, t)}{\partial t}
\end{gathered}
$$

Assuming cosine-based phasors, (7) and (8) can be rewritten as (9) and (10) and solving for $\mathrm{V}(\mathrm{z})$ and $\mathrm{I}(\mathrm{z})$ gives wave equations where $\gamma$ is the complex propagation constant. The traveling wave solutions can be written as (14) and (15). Finally, solving for the characteristic impedance $\mathrm{Z}_{\mathrm{o}}$ yields (16).

$$
\begin{gathered}
\frac{d V(z)}{d z}=-(R+j \omega L) I(z) \\
\frac{d I(z)}{d z}=-(G+j \omega C) V(z) \\
\frac{d^{2} V(z)}{d z^{2}}-\gamma^{2} V(z)=0 \\
\frac{d^{2} I(z)}{d z^{2}}-\gamma^{2} I(z)=0 \\
\gamma+j \beta=\sqrt{(R+j \omega L)(G+j \omega C)} \\
V(z)=V_{o}^{+} e^{-\gamma z}+V_{o}^{-} e^{\gamma z} \\
I(z)=I_{o}^{+} e^{-\gamma z}+I_{o}^{-} e^{\gamma z} \\
Z_{o}=\frac{R+j \omega L}{\gamma}=\frac{V_{o}^{+}}{I_{o}^{+}}=\frac{-V_{o}^{-}}{I_{o}^{-}}=\sqrt{\frac{R+j \omega L}{G+j \omega C}}
\end{gathered}
$$




\subsubsection{Transmission Lines and Characteristic Impedance}

The primary metric of a transmission line is characteristic impedance. For a lossless transmission line, the characteristic impedance can be simplified to be a real number as shown in (17). This expression is widely used in microwave engineering for deriving the characteristic impedance of many transmission line topologies. Fig. 2 shows several types of transmission line. Common planar transmission lines used in multilayer PCBs include microstrip, stripline, and coplanar waveguide. Standard characteristic impedances include $50 \Omega, 75 \Omega$, and $100 \Omega$.

$$
\begin{gathered}
R=G=0 \\
Z_{o}=\sqrt{\frac{L}{C}} \\
V=\frac{1}{\sqrt{L C}}
\end{gathered}
$$

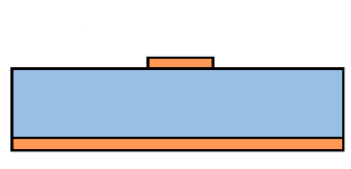

(a)

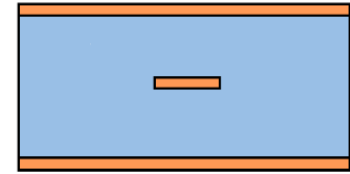

(b)

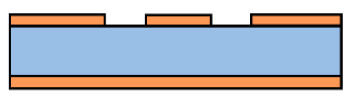

(c)

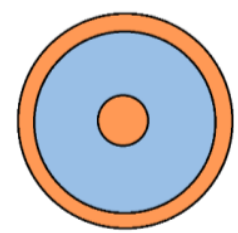

(d) (e)

Fig. 2. Various types of transmission lines. (a) microstrip (b) stripline (c) coplanar waveguide (d) coaxial (e) two wire. 


\subsubsection{Characteristic Impedance of a Coaxial Cable}

Due to radial symmetry, the characteristic impedance of a coaxial cable is mathematically friendly to derive and is discussed for demonstration. The capacitance per unit length (19) can be derived using Gauss's law by using a cylinder as the Gaussian surface. The inductance per unit length (20) can be derived using Ampere's law and using a circle as the Amperian loop. Substituting these equations into the characteristic impedance equation yields (21).

$$
\begin{gathered}
C=\frac{2 \pi \varepsilon_{r} \varepsilon_{o}}{\ln \left(\frac{b}{a}\right)} \quad\left(\frac{F}{m}\right) \\
L=\frac{\mu_{o}}{2 \pi} \ln \left(\frac{b}{a}\right) \quad\left(\frac{H}{m}\right) \\
Z_{o}=\sqrt{\frac{L}{C}}=\sqrt{\frac{\frac{\mu_{o}}{\frac{2 \pi}{2} \ln \left(\frac{b}{a}\right)}}{\frac{2 \pi \varepsilon_{r} \varepsilon_{o}}{\ln \left(\frac{b}{a}\right)}}}=\sqrt{\frac{\mu_{o}}{\varepsilon_{r} \varepsilon_{o}}} \frac{\ln \left(\frac{b}{a}\right)}{2 \pi}
\end{gathered}
$$

\subsection{Impedance Mismatch and Reflection Coefficient}

Transmission lines introduce the potential for reflected signals. The load of the transmission line defines a boundary condition for the ratio of voltage to current that must always be met. Since it takes a finite amount of time for the signal to propagate down the line and reach the load, this boundary condition does not have an effect until the signal is incident. Once the signal is incident on the load, a reflected wave must be propagated in the opposite direction to satisfy the boundary condition. Fig. 3 shows a transmission line with an arbitrary load. A reflection can occur at either end of the transmission line. 


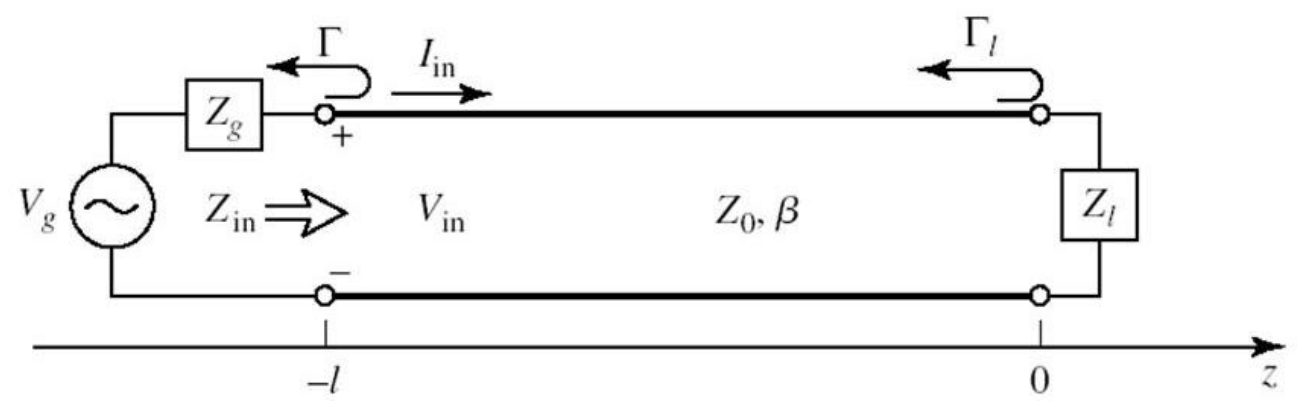

Fig. 3. Transmission line with arbitrary load $Z_{L}[3]$.

The reflection coefficient is defined as the ratio of the reflected voltage to the incident voltage and is a key parameter in RF systems. It can be expressed in terms of characteristic impedance and load impedance by solving for the ratio of voltage to current at the load. Since the origin is defined at the load, this is when $\mathrm{z}=0$ as shown in (22). Solving for the ratio of reflected voltage to incident voltage yields the reflection coefficient (23).

$$
\begin{gathered}
V(z)=V_{o}^{+} e^{-j \beta z}+V_{o}^{-} e^{j \beta z} \\
I(z)=\frac{V_{o}^{+}}{Z_{o}} e^{-j \beta z}-\frac{V_{o}^{-}}{Z_{o}} e^{j \beta z} \\
Z_{L}=\frac{V(0)}{I(0)}=\frac{V_{o}^{+}+V_{o}^{-}}{V_{o}^{+}-V_{o}^{-}} Z_{o} \\
V_{o}^{-}=\frac{Z_{L}-Z_{o}}{Z_{L}+Z_{o}} V_{o}^{+} \\
\Gamma=\frac{V_{o}^{-}}{V_{o}^{+}}=\frac{Z_{L}-Z_{o}}{Z_{L}+Z_{o}}
\end{gathered}
$$


From this equation, one can see that reflections occur when the impedance of a transmission line does not match the impedance of the load. Thus, to achieve maximum power transfer, it is required that the impedance of the generator and load match the characteristic impedance of the transmission line. In a multilayer PCB, the via presents a discontinuity in impedance and can cause reflections.

\subsection{Time-Domain Reflectometry}

A common tool for impedance matching is the smith chart, however, the smith chart only describes the impedance looking into a port and does not describe the physical location of where impedance mismatch occurs. To determine the physical location, one can use time domain reflectometry (TDR) which utilizes a concept similar to radar. In this technique, a step or an impulse is propagated through the network and the reflection coefficient vs time is measured.

If the load impedance is higher than the characteristic impedance of the transmission line, the reflected wave will have a positive amplitude, and constructively interfere with the incident wave. Thus, the reflection coefficient will show an increase once the reflected wave reaches the port. If the impedance is lower than the characteristic impedance of the transmission line, the reflected wave will have a negative amplitude and destructively interfere with the incident wave. This will show a decrease in reflection coefficient once the reflected wave reaches the port. Based on the time delay and the speed of propagation, one can determine the location at which a reflection is induced and whether the junction is higher or lower in impedance. 
Fig. 4 shows the bounce diagram of a two-layer PCB with a via. The reflection from the via reaches the input port before the reflection caused by the load. Using the smith chart, one could not differentiate between the two reflections. Time-domain reflectometry yields insight to which junction causes the reflection and its impedance.

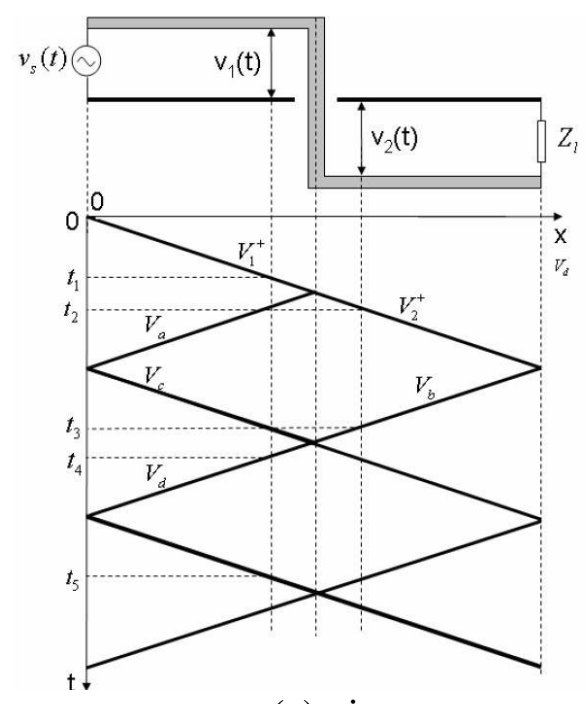

(a) via

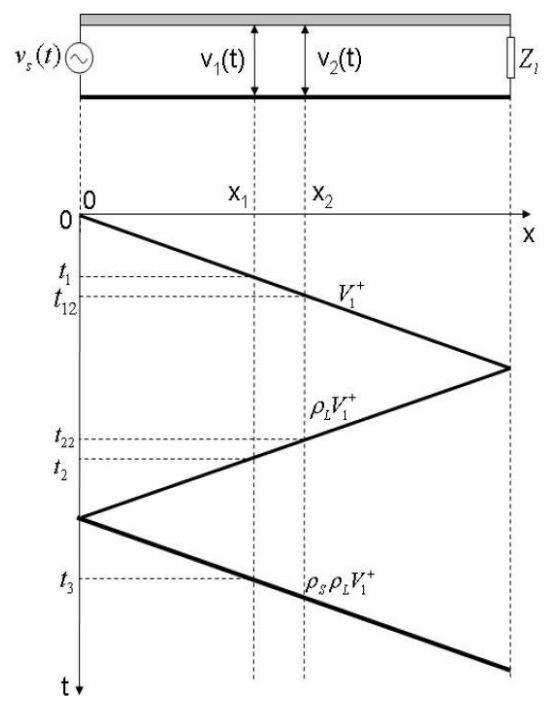

(b) without via

Fig. 4. Bounce diagram of a microstrip with and without a via [4].

\subsection{S-Parameters}

The scattering parameters (S-parameters) are foundational to microwave and RF engineering. It is a matrix that describes how voltage is scattered through a network. The matrix element, $\mathrm{S}_{\mathrm{ba}}$ is defined as the ratio of the voltage leaving port $\mathrm{b}$ to the voltage entering port a when all ports beside port a are terminated with a matched load. For example, consider the two-port network depicted in Fig. 5. Allow b to denote the voltage leaving the port and a to denote the voltage entering the port. $\mathrm{S}_{11}$ is by definition the reflection coefficient for port 1 (24), and $S_{21}$ is the transmission coefficient for port 1 
(25). Similarly, $\mathrm{S}_{22}$ is the reflection coefficient for port 2 (26), and $\mathrm{S}_{12}$ is the transmission coefficient for port 2 (27).

$$
\begin{gathered}
S_{11}=\Gamma=\frac{b_{1}}{a_{1}} \\
S_{21}=\tau=\frac{b_{2}}{a_{1}} \\
S_{22}=\frac{b_{2}}{a_{2}} \\
S_{12}=\frac{b_{1}}{a_{2}}
\end{gathered}
$$

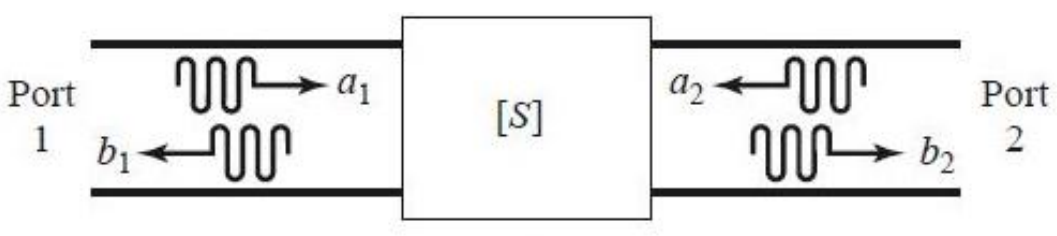

(a)

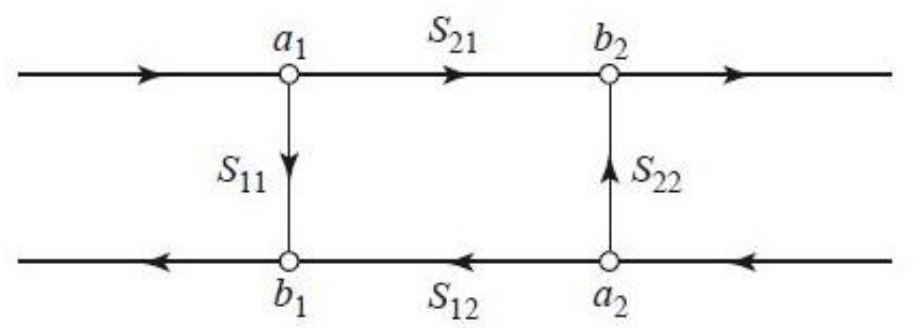

(b)

Fig. 5. Two-port network and equivalent flow diagram [3]. 


\section{MULTILAYER PCB MANUFACTURING PROCESS}

Prior to starting a design, it is important to understand the limitations presented by the manufacturing process. The fabrication of a multilayer PCB is quite involved, and the general process is described below [5].

\subsection{General Multilayer PCB Manufacturing Process}

The process starts with the innermost layer. The design is first printed on a transparent film that is used for photo-etching the copper clad. The copper clad laminate is then uniformly coated with a light sensitive film called the photoresist. Since each layer of the PCB needs to line up, precise alignment holes are punched into the films and laminates. The copper clad laminate is aligned with the film containing the design and is then exposed to intense UV light which hardens the photoresist. The areas that are covered by the hardened photoresist are where the copper will be. The exposed copper that is not covered by the hardened photoresist will be dissolved away using an alkaline solution. Once the unwanted copper is dissolved, the hardened photoresist is removed using a chemical solution and the innermost layer is ready to be bonded to its surrounding layers.

To adhere two layers together, a laminate impregnated with uncured epoxy resin called prepeg is used. The layers and prepeg are tightly sandwiched in a press and heated in an oven to cure the epoxy resin. This bonds the layers together. An X-ray drill is then used to drills vias precisely at the center of the via pads. The via holes are then plated with copper using electroplating. The photoetching, layer bonding, drilling, and plating process is repeated for each layer until the board is complete [5]. 


\subsection{High Frequency Considerations}

Due to transmission line effects, additional considerations must be made to maintain performance at high frequency. This includes material selection, layer bonding technique, and via dimensions.

\subsubsection{Material Selection and PTFE}

The primary distinction is the choice of substrate materials. In a transmission line, the signal propagates as an electromagnetic wave between the signal trace and the ground plane. Thus, the material properties of the substrate heavily define the characteristics of the transmission line. Key parameters include dielectric constant and loss tangent. Conventional materials used in low frequency applications such as FR-4 are mechanically rigid and inexpensive but are lossy at high frequency. Therefore, special substrate materials such as polytetrafluroethylene (PTFE) are preferred for RF application.

PTFE is widely used in microwave circuits for its low dielectric constant and low loss. However, PTFE has several drawbacks that can make it difficult to use in a multilayer PCB.

PTFE laminates have a relatively high coefficient of thermal expansion (CTE). Manufacturers offer laminates which are structurally reinforced with fiberglass, glass fiber, or ceramic fillers. This helps with the thermal expansion along the plane of the laminate, but the thickness of the laminate can still increase with temperature. This can be problematic for plated through holes, as they can fatigue and crack after many temperature cycles [6]. 
PTFE laminates are also relatively expensive. Therefore, it is sometimes desirable to mix PTFE with less expensive materials. Hybrid multilayer PCBs made from both PTFE and FR-4 are ideal for boards that contain both DC and RF signals. It is important to choose materials with a similar CTE to prevent mechanical issues such as delamination. To prevent delamination and cracking of plated through holes, one should choose laminates with a CTE closest to that of copper.

In addition, PTFE is a relatively soft material which requires special drilling and plating techniques for the vias, thus a PCB manufacturer who specializes with PTFE should be used [7].

\subsubsection{Bonding and Stack-Up}

The stack-up is the order in which the laminates are bonded together. For low frequency applications, a prepeg layer is used for bonding. For high frequency applications, this prepeg presents a discontinuity in the material properties and must be considered in the electrical design as it can change the characteristic impedance of a transmission line. There are three ways to bond layers in a multilayer PCB, each with a tradeoff between cost and performance. These configurations are shown in Fig. 6, and the tradeoffs are summarized in Table 1.

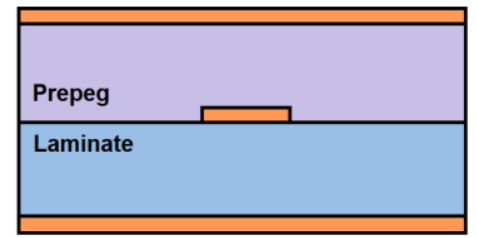

(a)

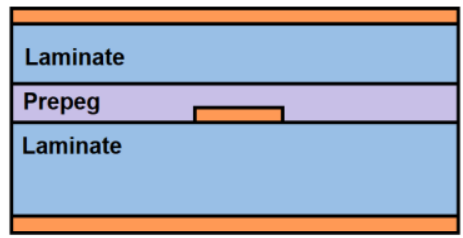

(b)

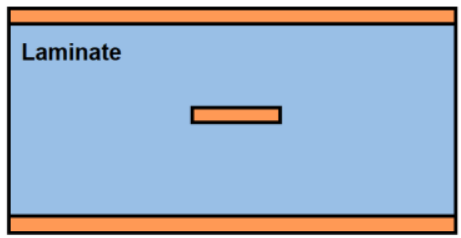

(c)

Fig. 6. PTFE stack-up options. (a) PTFE \& Prepeg (b) Two PTFE with Prepeg Film (c) All PTFE (Fusion Bonding). 
Table 1

PTFE Stack-Up Summary

\begin{tabular}{|c|c|c|c|}
\hline Stack-Up & Performance & Cost & Fabrication Support \\
\hline PTFE \& Prepeg & Lowest & Lowest & Common \\
\hline Two PTFE with Prepeg Film & Medium & Medium & Common \\
\hline All PTFE (Fusion Bonding) & Highest & Highest & Uncommon \\
\hline
\end{tabular}

The most inexpensive way to bond layers is to combine a PTFE laminate and a prepeg laminate. This way, the stripline is half PTFE and half prepeg. Prepeg is cheaper than PTFE laminates and is typically more lossy. This means the stripline would consist of two different materials, and the characteristic impedance of the transmission line would have to be designed accordingly. A comparison between different prepeg materials is shown in Table 2 [8].

Table 2

Common Bonding Materials

\begin{tabular}{|l|c|c|}
\hline \multicolumn{1}{|c|}{ Bonding Material } & $\begin{array}{c}\text { Dielectric Constant } \\
\left(\boldsymbol{\varepsilon}_{\mathbf{r}}\right)\end{array}$ & $\begin{array}{c}\text { Loss Tangent } \\
(\tan \boldsymbol{\delta})\end{array}$ \\
\hline FR-4 (industry standard) & 4.5 & 0.0180 \\
\hline Fluorinated Ethylene Propylene (FEP) & 2.1 & 0.0010 \\
\hline Chloro-Fluorocopolymer & 2.3 & 0.0030 \\
\hline Ceramic-filled Hydrocarbon Prepreg & 3.9 & 0.0040 \\
\hline Liquid Crystalline Polymer (LCP) & 2.9 & 0.0025 \\
\hline
\end{tabular}

Alternatively, one can use two PTFE laminates and bond them using a thin prepeg film. The thickness of a prepeg film is typically 1.5 mil [8]. If the PTFE is much thicker 
than the prepeg film, the PTFE properties dominate and the prepeg film is negligible. This allows for more consistent thickness and a low loss material to be used on both sides of the stripline. This technique requires the entire copper plane to be removed from one laminate and the design to be etched in the other laminate to make the other half of the stripline. This is the most practical way to achieve a low loss stripline transmission line, but it is more expensive since two PTFE laminates are needed.

Lastly, one can also reflow PTFE laminates together. This is a nonconventional method, but it is the most ideal since this allows for a pure, homogenous stripline substrate. In this process, the copper plane is removed from one laminate, and the substrate of both laminates are melted and bonded back during the cooling process. While ideal, it is uncommon for manufacturers to support this method [8].

\subsubsection{Vertical Connections}

The via is a vertical connection between layers and is the primary reason multilayer PCBs are difficult to use at high frequency. This is because they present a discontinuity in impedance which can result in reflections. The dimensions of the via can be optimized to minimize the reflection caused by this vertical junction. Fig. 7 shows the different parts of a via. There are several types of vias. A through-hole via goes through all the layers and connects the outer most layers. A blind via connects an outer layer to an inner layer. A buried via connects inner layers and is not visible from the outside. 


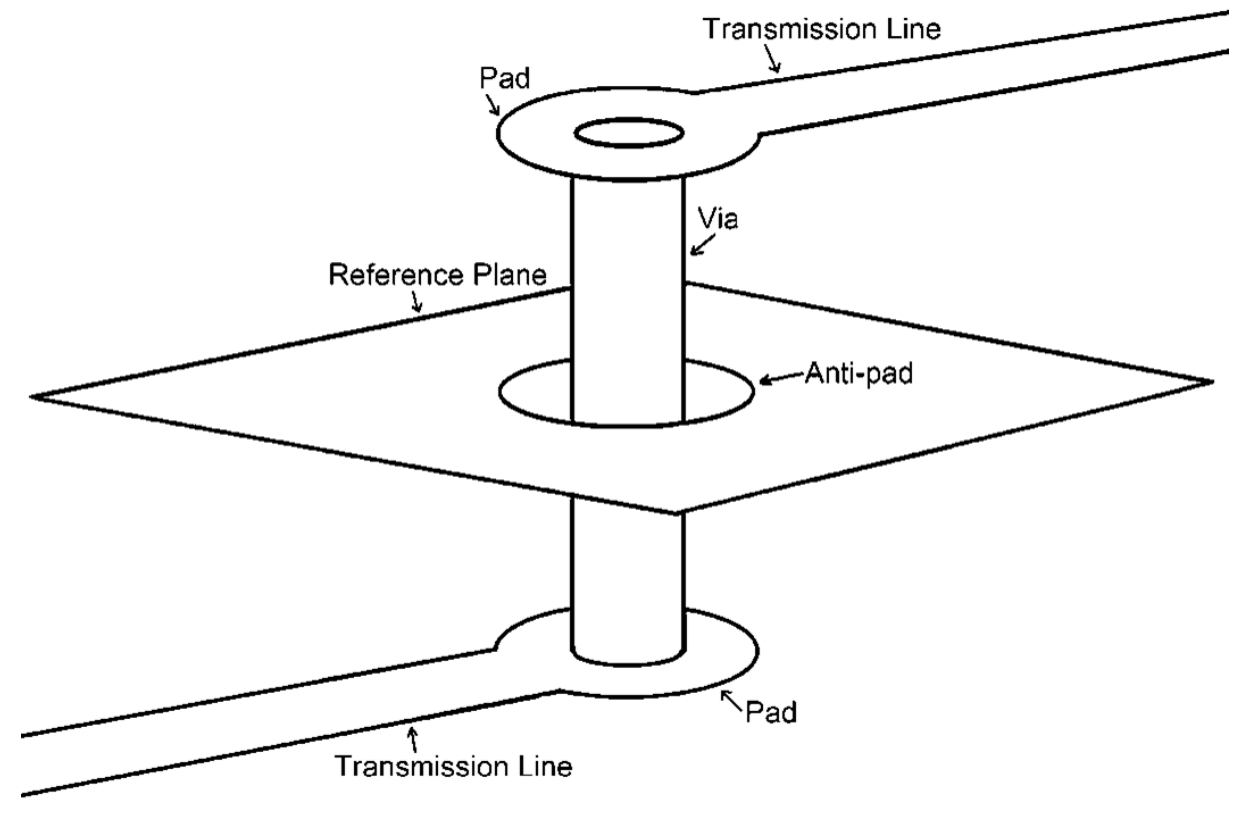

Fig. 7. Via hole anatomy. 


\section{LITERATURE REVIEW}

\subsection{Lumped Element Model}

Many attempts have been made to model a via, but it is difficult to characterize the vertical junction with a closed form expression. A popular lumped element model from the book "High Speed Digital Design: A Handbook of Black Magic" by Martin Graham [9] is shown in Fig. 8. In this book, Graham states that because a via is a small structure, it can be modeled as lumped elements as opposed to a transmission line. While it is common knowledge among RF engineers that a via introduces parasitic series inductance, this model includes shunt capacitance as well. This shunt capacitance accounts for the capacitance between the plated through hole (PTH) and the reference plane. Several IEEE papers reference this model [10]-[13].

$$
C=\frac{1.41 \varepsilon_{r} h_{c} d_{c}}{D_{c}-d_{c}}
$$

In (28), $\mathrm{D}_{\mathrm{c}}$ is the diameter of antipad, $\mathrm{d}_{\mathrm{c}}$ is the diameter of pad, $\mathrm{h}_{\mathrm{c}}$ is the thickness of $\mathrm{PCB}$, and $\mathrm{C}$ is the parasitic capacitance in $\mathrm{pF}$. All the dimensions are in inches.

$$
L=5.08 h_{L}\left[\ln \left(\frac{4 h_{L}}{d_{L}}\right)+1\right]
$$

In (29), $h_{L}$ is the length of the via, $d_{L}$ is the diameter of the via, and $L$ is parasitic inductance in $\mathrm{nH}$. All the dimensions are in inches.

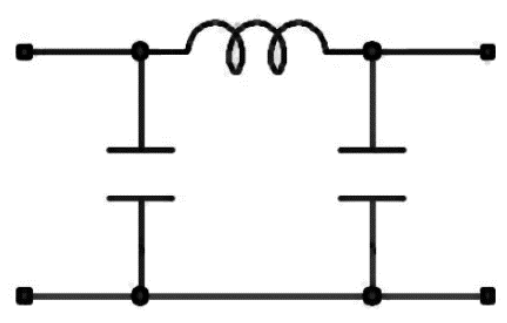

Fig. 8. Lumped element model of a via. 


\subsection{EM Simulation Optimization}

In addition to the lumped element models, several works [11], [15] report the use of EM simulation to optimize the via. In these works, via dimensions are chosen, and a parametric sweep is done on each dimension of the via. This includes via hole diameter, pad diameter, and antipad diameter. By sweeping these variables, one can optimize the reflection coefficient and minimize the impedance mismatch. Optimization using parametric sweep in EM simulation seems to be the most effective technique for choosing via dimensions, but this process is also time consuming since EM simulation is relatively slow and is likely to require several iterations. Thus, it is important to know how changing each parameter will affect the impedance of the junction. A technical paper published by John Coonrod of Rogers Corporation titled "Influence of Through Hole Vias on PCB RF Performance" has an excellent summary of how changing each dimension effects the impedance of the via [16]. This summary is shown in Table 3. These guidelines allow the designer to iteratively tune the impedance of the via to $50 \Omega$.

Table 3

Impedance Optimization Guidelines

\begin{tabular}{|c|c|c|}
\hline Parameter & Increase Parameter & Decrease Parameter \\
\hline Via Hole Diameter & Capacitive & Inductive \\
\hline Pad Size & Capacitive & Inductive \\
\hline Copper Plating Thickness & Capacitive & Inductive \\
\hline Dielectric Constant & Capacitive & Inductive \\
\hline Anti-Pad Size & Inductive & Capacitive \\
\hline Via Hole Length & Inductive & Capacitive \\
\hline
\end{tabular}


One IEEE paper [13] mentions that a buried via has more shunt capacitance due to there being a reference plane on both sides of the via. This means a through hole, blind, and buried via would need to have slightly different geometries to achieve minimal reflection. It was also suggested that if less capacitance was needed, a hole in either reference plane could be made to reduce shunt capacitance.

Several papers report how other parasitic effects caused by vias can be mitigated. One IEEE paper reports that a larger via hole diameter reduces insertion loss due to a larger conductor cross-section [19]. Lastly, several IEEE papers [11], [15], [17]-[19] report on the use of grounding vias to shield the signal via. According to the simulations, this helped prevent radiation leakage into the reference planes and reduced power loss. These design rules are summarized in Table 4.

Table 4

Guidelines for Other Parasitic Effects

\begin{tabular}{|c|c|}
\hline Design Rule & Benefit \\
\hline Use Larger Via Hole Diameters & Less Insertion Loss \\
\hline Use Shorter Vias & Less Stub Resonance Effects \\
\hline More Shielding Vias & Less Radiation Leakage \\
\hline
\end{tabular}

\subsection{Shielding Vias and Coaxial Via}

The via also presents the issue of crosstalk and energy leakage. Anytime there is a break in the return current path, electromagnetic energy is not guided along the transmission line and is radiated away. A vertical transition from one layer to another is problematic because of the change in the reference planes. With this transition, the return 
current path is not clear and electromagnetic energy can leak between the reference planes.

Several IEEE papers [11], [15], [17]-[19] study the effect of shielding vias. Shielding vias surround the signal via and connect the reference planes of each layer. This creates a path for return current and helps guide the electromagnetic energy. Without shielding vias, electromagnetic energy is able to radiate and excite modes between the reference planes. EM simulation shows that without the vias, the insertion loss has a sharp roll-off at the first resonant frequency from mode excitation between reference planes. This can also result in cross talk with nearby transmission lines. With 8 shielding vias, the resonant frequency is removed, and the insertion loss is minimal [15]. A simulated E-field magnitude plot also showed that the electromagnetic field is well contained in the shielding vias.

One paper reports on a technique that can be used to measure the capacitance of the via and suggested that if the number of reference planes is increased, the vertical transition can resemble a coaxial cable. The measured capacitance was close to the calculated value for a coaxial cable [12].

\subsection{Nonconventional Vias}

Outside of the traditional via, several experiments on nonconventional vias have been reported. One IEEE paper [20] tested several different shapes of via hole pad. Instead of a simple circle, shapes such as a bottleneck, taper, and no taper were tested. After the designs were optimized in EM software, measurements showed that each shape yielded 
$3 \mathrm{~dB}$ to $5 \mathrm{~dB}$ improvement in return loss when compared to a traditional via. However, measurements were only shown up to $3 \mathrm{GHz}$.

Another paper [21] tested a row of vias to connect two microstrips. The design was optimized using HFSS, and the simulation matched measurements up to $8 \mathrm{GHz}$.

Measurements showed a return loss of at least $-20 \mathrm{~dB}$ up to $8 \mathrm{GHz}$, and an insertion loss of less than $-1 \mathrm{~dB}$ up to $8 \mathrm{GHz}$.

Lastly, two unique papers [22], [23] used 3D printing to construct a via. This allowed for nonplanar geometries to be tested. One paper tested a tapered ramp for a microstrip to stripline transition. Both 3D printed vias had limited performance. 


\section{DESIGN AND SIMULATION}

Since many combinations of planar transmission lines, substrate materials, and substrate thicknesses are possible, the work in this thesis is aimed to demonstrate a general process for maintaining high frequency performance in multilayer PCBs for any application. The transmission lines for these simulations are microstrip and stripline, and simulations of a 1-level, 2-level, and 3-level transition are shown.

\subsection{PCB Stack-Up}

The dimensions of the simulated PCB are chosen to be realistic for manufacturing. The substrate used is Rogers Duroid 6006 which has a dielectric constant of 6.15 and a loss tangent of 0.0018 . A 3-level drop requires 7 laminates. Thicker substrates are preferred by PCB fabricators when more layers are used. The layer thicknesses used include $20 \mathrm{mil}$ for the uppermost layer and $30 \mathrm{mil}$ for the other layers. This allows for a $50 \Omega$ microstrip to have a width of 30 mils and a $50 \Omega$ stripline to have a width of 18 mils. Lastly, an air cavity height of 140 mils is chosen.

\subsection{Simulation Set-Up}

For EM simulations, Ansys HFSS 15.0 was used. Wave ports were used to excite the microstrip and stripline, and the recommended port sizing as published by Ansys was used. Port widths of 8 times the transmission line widths are chosen for both ports. The conductors are modeled as zero thickness, 2D planar structures. The boundary chosen is perfect E-field. The substrate is defined as Rogers Duroid 6006 from the HFSS material library and fusion bonding is assumed for layer bonding. 


\subsection{Via Stitching}

In a multilayer PCB, it is necessary to connect the reference planes near the signal via, otherwise the space between each reference plane forms a cavity that can be excited (assuming the reference planes are connected elsewhere). Fig. 9 shows a microstrip to stripline transition whose reference planes are connected at the edges. Although this design yields a return loss of less than $-20 \mathrm{~dB}$ up to $4.5 \mathrm{GHz}$, the first resonant frequency of this 1-level transition is at $5.9 \mathrm{GHz}$ as shown in Fig. 10 .

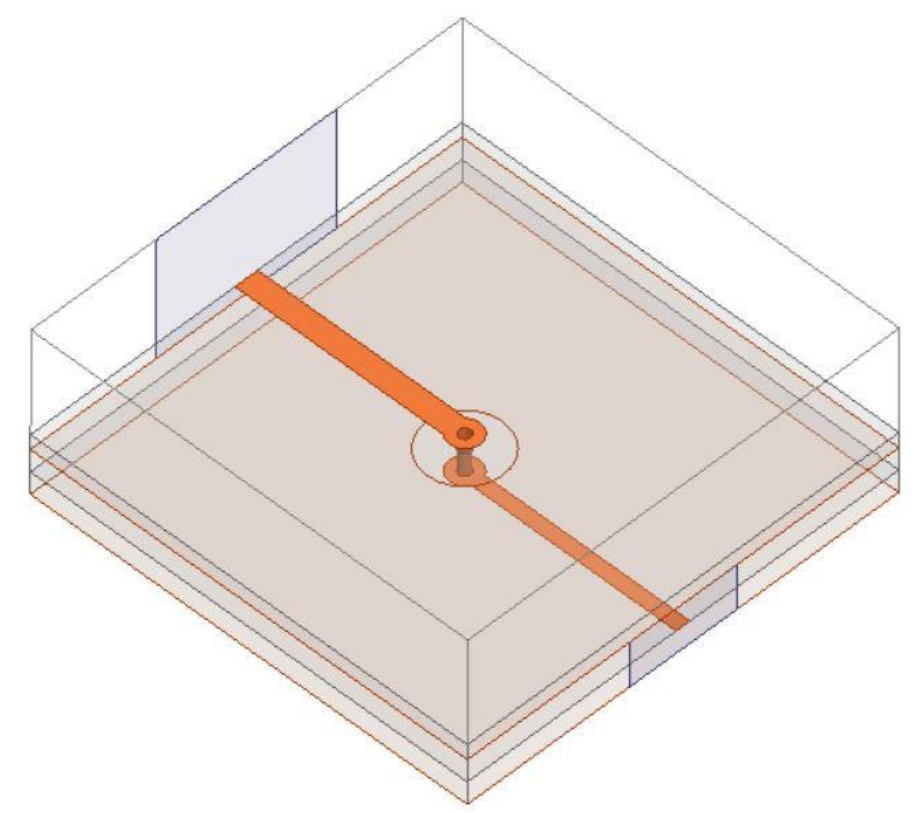

Fig. 9. 1-level transition with no stitching vias. The reference planes are connected at the edges.

Using the E-field magnitude plot shown in Fig. 11, this resonance is confirmed to be from the cavity formed between the reference planes. The larger the cavity, the lower this resonant frequency will be. The size of this cavity is chosen such that the first resonant frequency interferes with the passband of the filter that will be cascaded in a later section to demonstrate the effectiveness of the design. If the reference planes are not connected, 
there will be no cavity resonance effect, but the signal will be radiated away resulting in significant insertion loss.

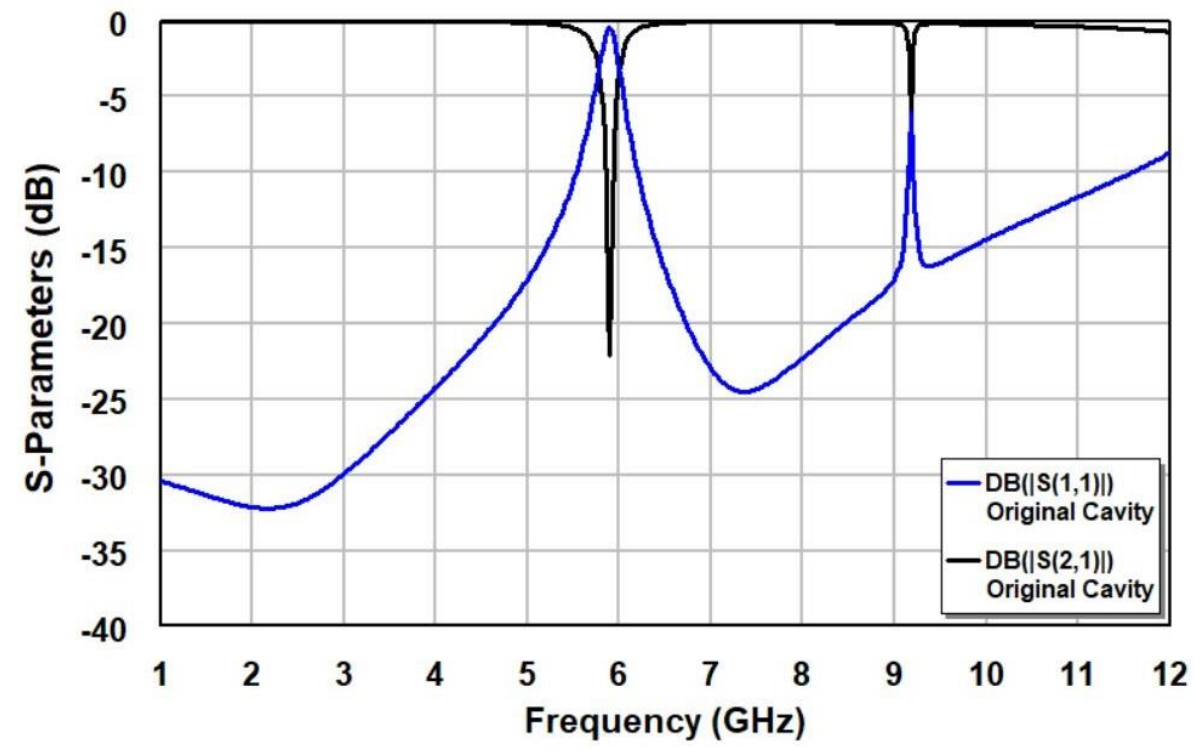

Fig. 10. S-parameters of the 1-level transition with no stitching vias.
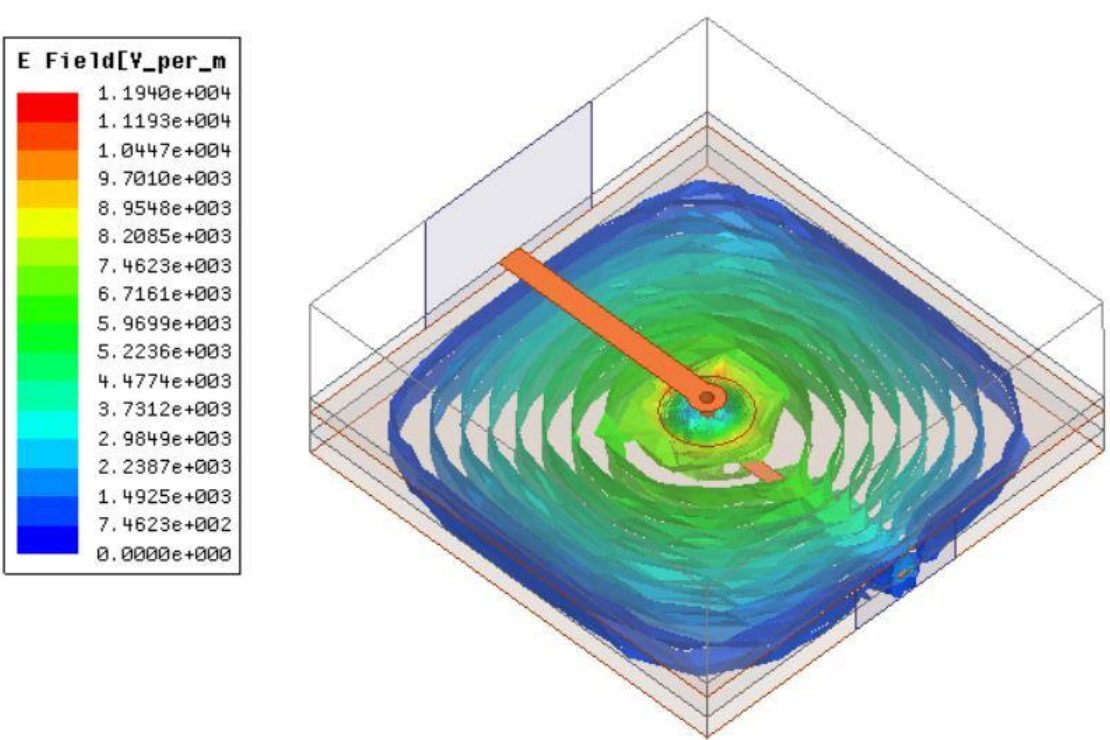

Fig. 11. E-field magnitude plot at $5.9 \mathrm{GHz}$. Without stitching vias, modes can be excited between the reference planes. 
In addition, the 3-level transition with no stitching vias shown in Fig. 12 was simulated. This transition has 3 cavities that can be excited, causing the first resonant frequency to become broader as shown in Fig. 13. Fig. 14 shows the E-field magnitude plot at the resonant frequency.

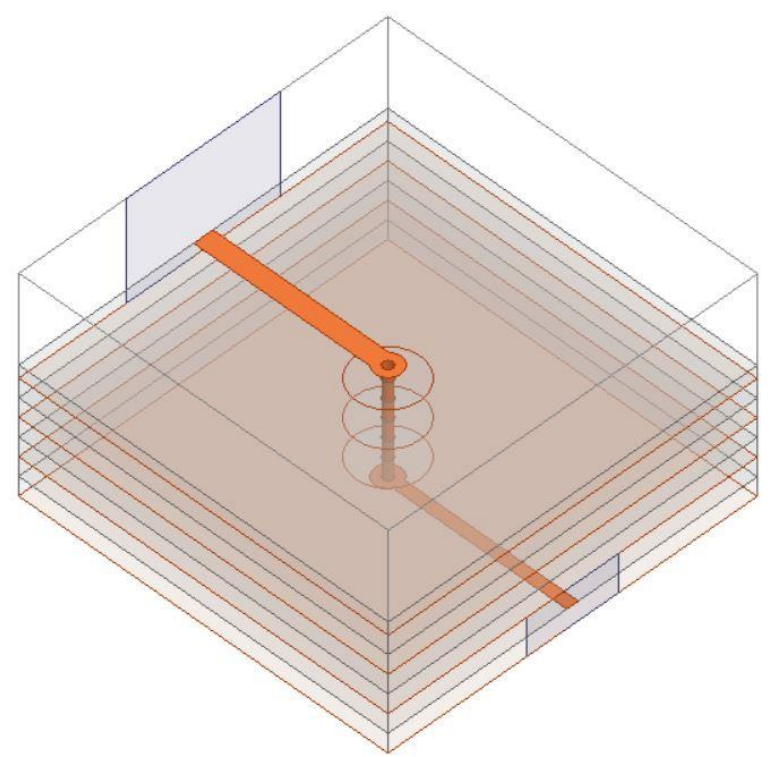

Fig. 12. 3-level transition with no stitching vias.

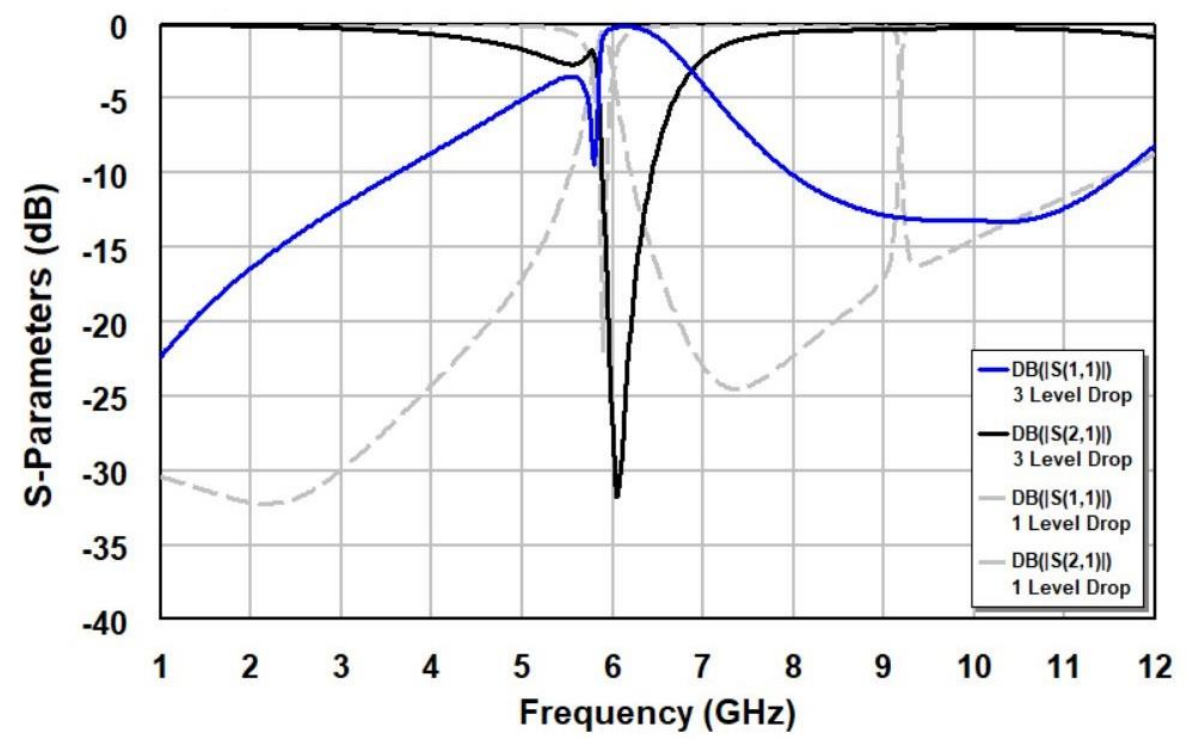

Fig. 13. S-parameters of the 3-level transition with no stitching vias. The resonant frequency becomes much broader in comparison to the 1-level transition. 

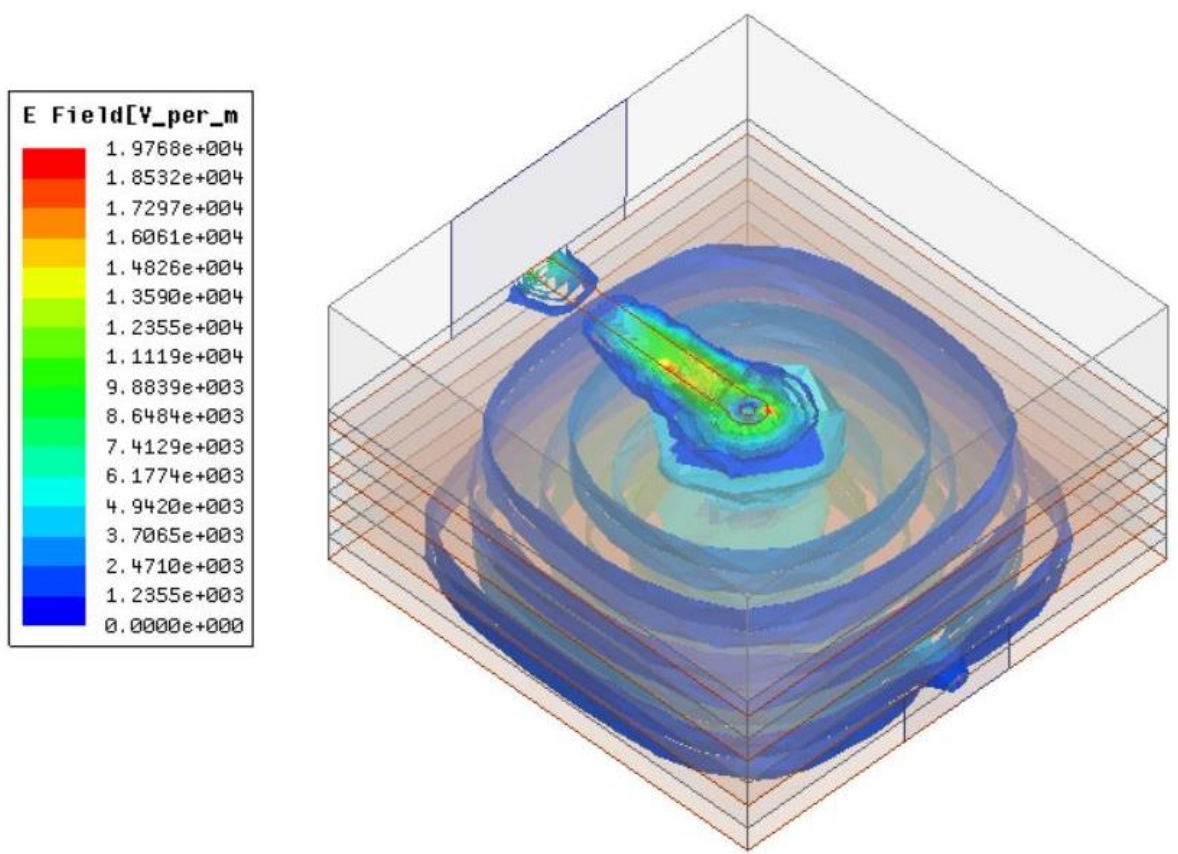

Fig. 14. E-field magnitude plot of a 3-level transition with no stitching vias at $5.9 \mathrm{GHz}$.

In order to mitigate this effect, via stitches are placed near the signal via for shielding and to offer a closer return current path. To determine how many stitching vias were needed, the configurations shown in Fig. 15 were simulated. This includes 2, 4, 6, and 8 stitching vias. With each via equidistant from each other, the most vias possible were 8 since additional vias would then intersect with the stripline. 

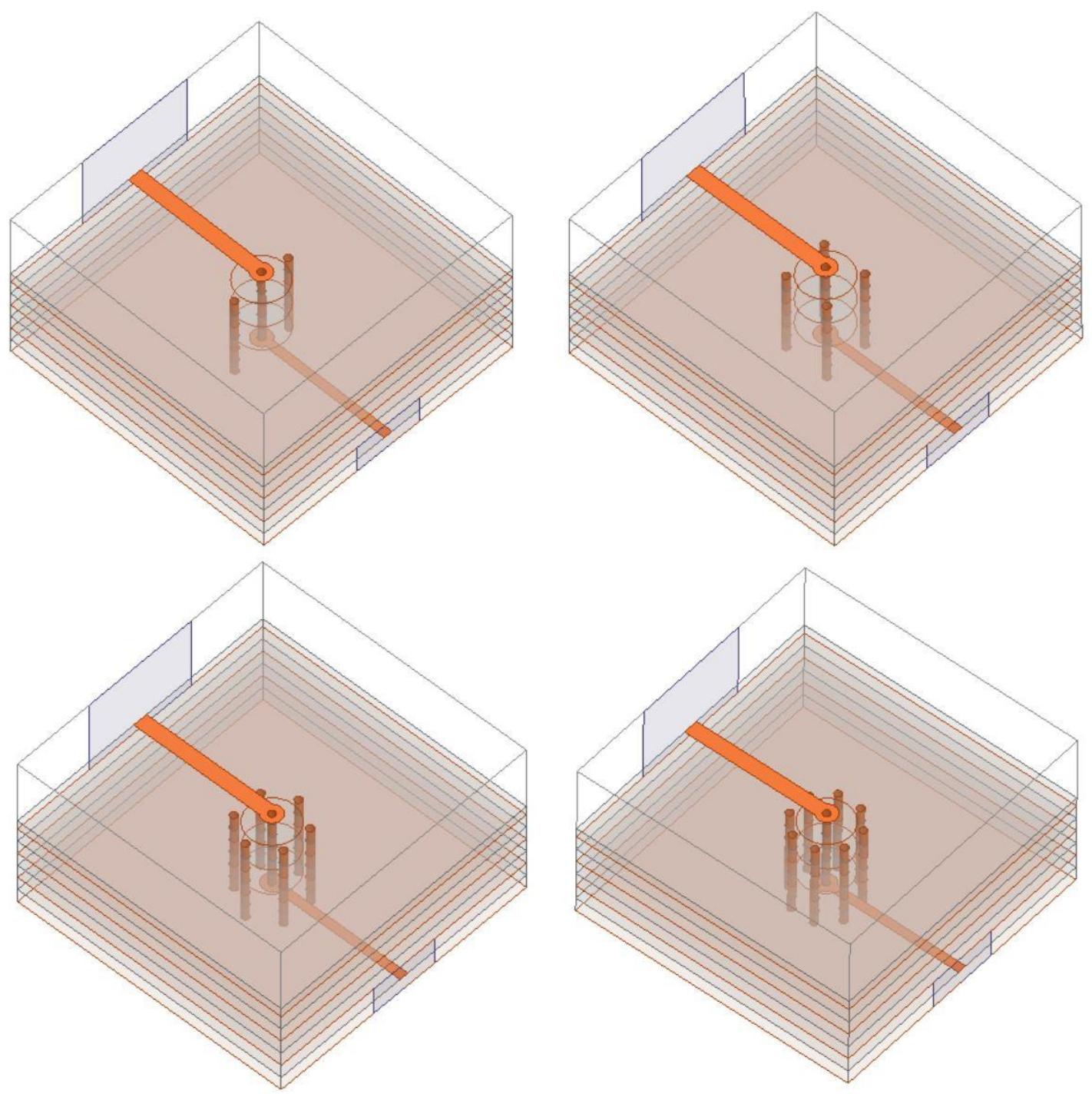

Fig. 15. 2, 4, 6, and 8 stitching vias on a 3-level transition. 


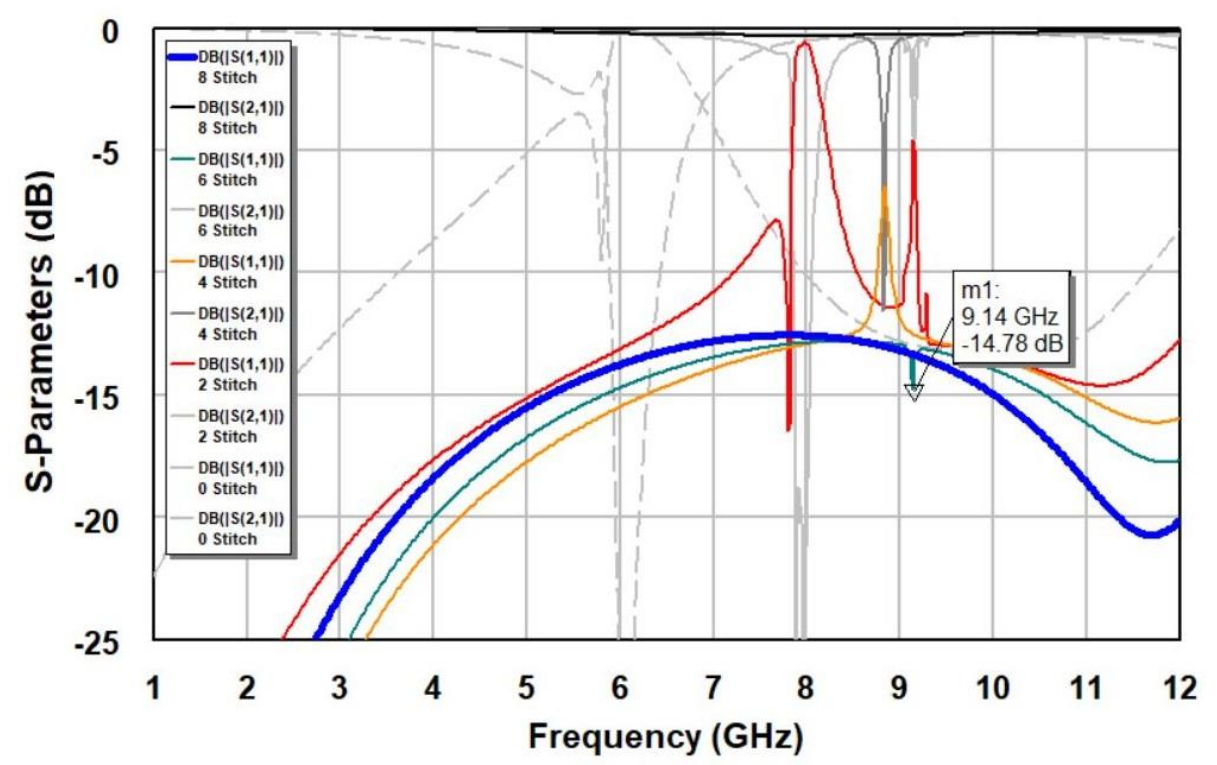

Fig. 16. S-parameters of 0, 2, 4, 6, and 8 stitching vias.

From Fig. 16, one can see that the shielding effectiveness is related to the distance between vias. As expected, more stitching vias progressively dampen the first resonant frequency. Six stitching vias effectively blocked any cavity resonance up to $9.14 \mathrm{GHz}$. Eight stitching vias effectively blocked any cavity resonance up to $13.6 \mathrm{GHz}$. A summary of the effectiveness of the stitching vias is shown in Table 5. In Fig. 17, one can see that the E-field is now well contained in the via shield.

Table 5

Via Stitch Spacing

\begin{tabular}{|c|c|c|}
\hline Number of Stitch Vias & Via Spacing & First Cavity Resonance \\
\hline 2 Stitch Vias & $110 \mathrm{mils}$ & $7.6 \mathrm{GHz}$ \\
\hline 4 Stitch Vias & $73.3 \mathrm{mils}$ & $8.84 \mathrm{GHz}$ \\
\hline 6 Stitch Vias & $47.5 \mathrm{mils}$ & $9.14 \mathrm{GHz}$ \\
\hline 8 Stitch Vias & $32.8 \mathrm{mils}$ & $13.6 \mathrm{GHz}$ \\
\hline
\end{tabular}



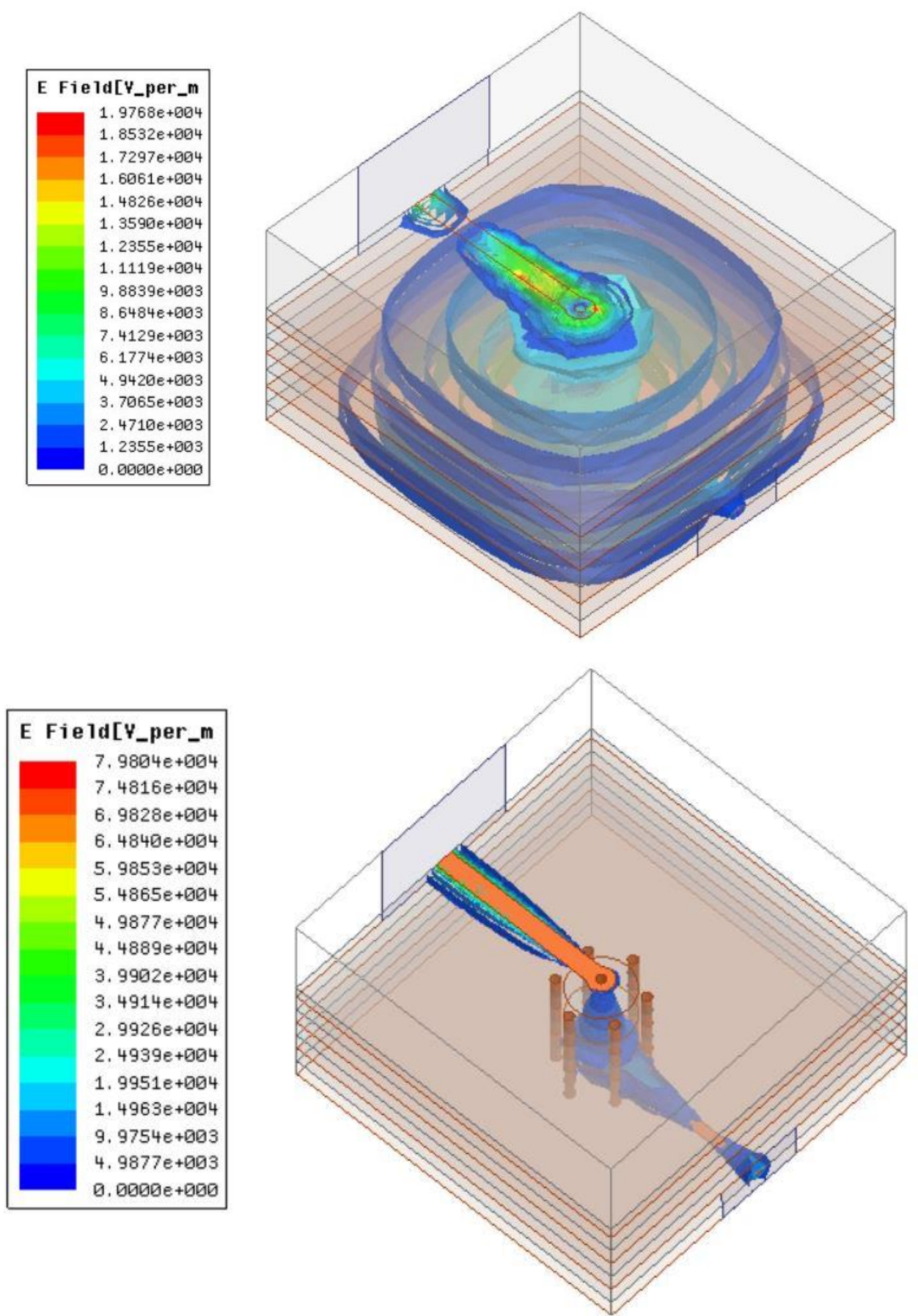

Fig. 17. E-field magnitude plot of a 3-level transition at $5.9 \mathrm{GHz}$ with and without stitching vias. With 6 stitching vias, the E-field is well contained. 


\subsection{Impedance Matching the Via}

In addition to via stitching, it is critical to choose dimensions such that the impedance of the via matches that of the transmission lines. To do this, time-domain reflectometry in HFSS is used, and the guidelines in Table 3 are followed. Since the substrate thickness and dielectric constant are fixed, there are only four parameters that can be tuned to control the impedance. This includes via radius, microstrip pad radius, stripline pad radius, and antipad radius. Arbitrarily choosing these dimensions can result in a low impedance via or a high impedance via and the tuning process for both are shown.

\subsubsection{Low Impedance Via}

As shown in Fig. 18, a low impedance via will show as a capacitive dip in the TDR plot. This results in poor return loss as shown in Fig. 19. To fix this, one can either decrease the via diameter, increase antipad radius, decrease microstrip pad radius, or decrease stripline pad radius.

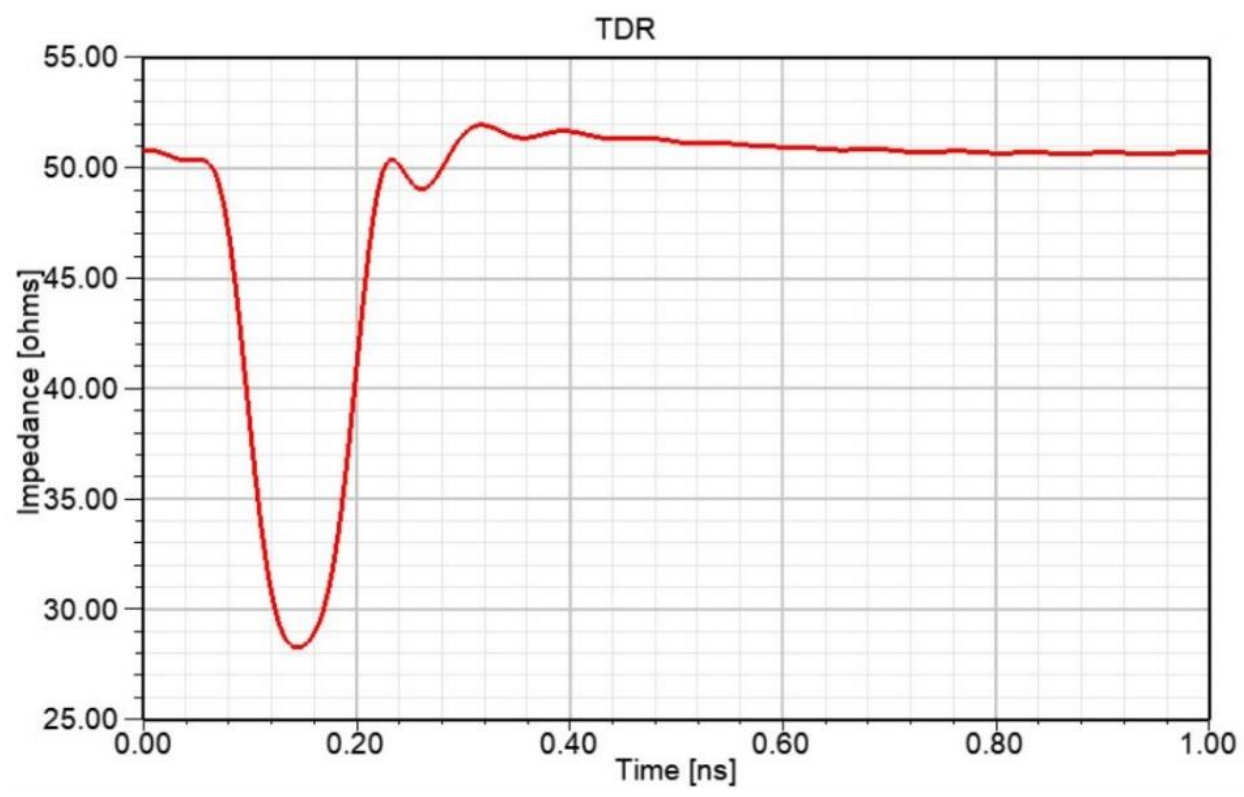

Fig. 18. TDR plot for a low impedance via. 


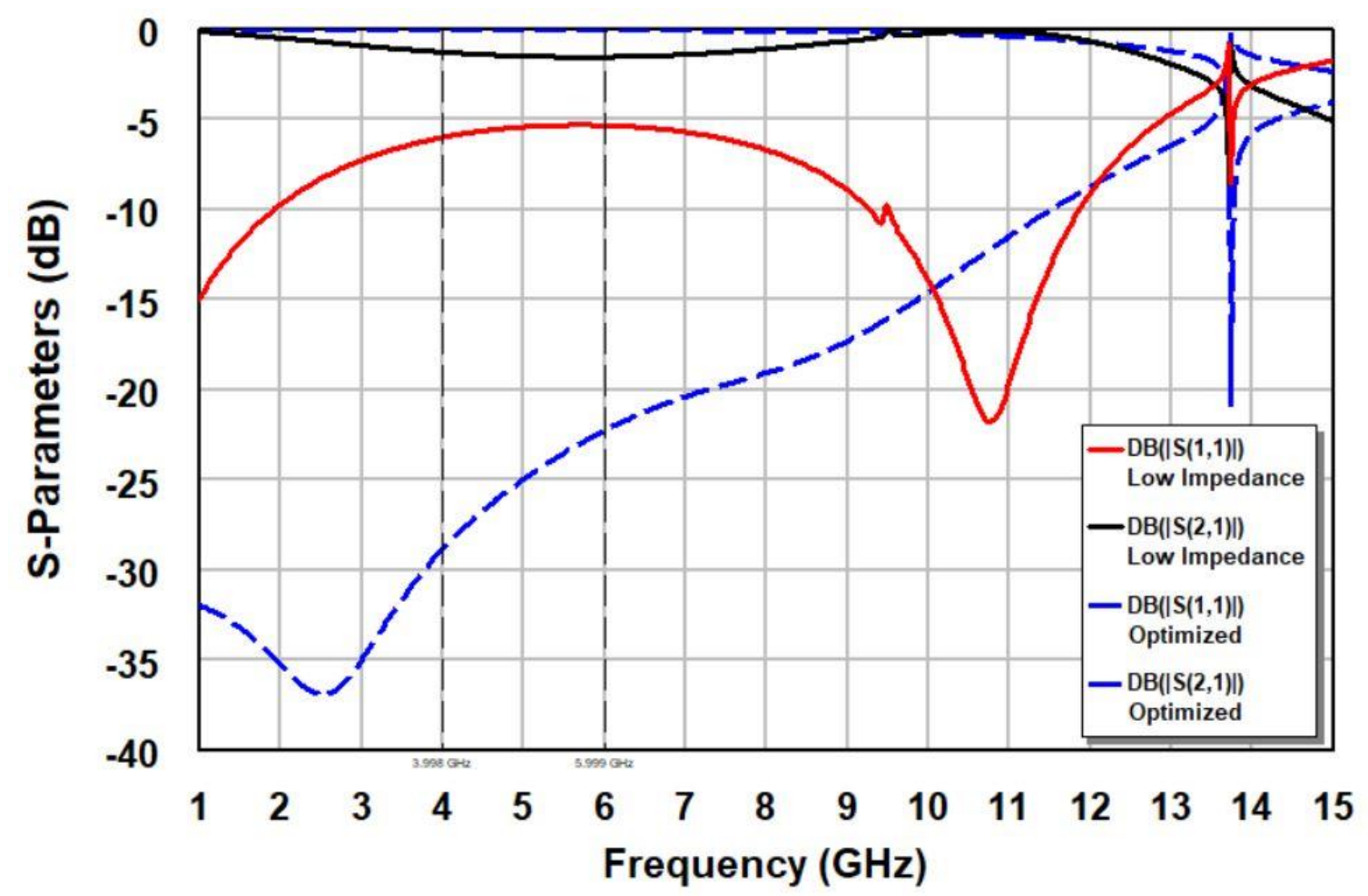

Fig. 19. S-parameters of the low impedance via and the optimized via. Tuning the dimensions can significantly improve return loss.

\subsubsection{High Impedance Via}

As shown in Fig. 20, a high impedance via will show as an inductive spike in the TDR plot. This results in poor return loss as shown in Fig. 21. To fix this, one can either increase the via diameter, decrease antipad radius, increase microstrip pad radius, or increase stripline pad radius. An advantage to using TDR over smith chart is that TDR describes the physical location of the impedance mismatch. Because the junction is so small and the rise-time of the signal is too slow, one cannot use TDR to tune each variable. Rather, the dip or spike in the TDR is blended together into one hump and one cannot differentiate between the different junctions. 


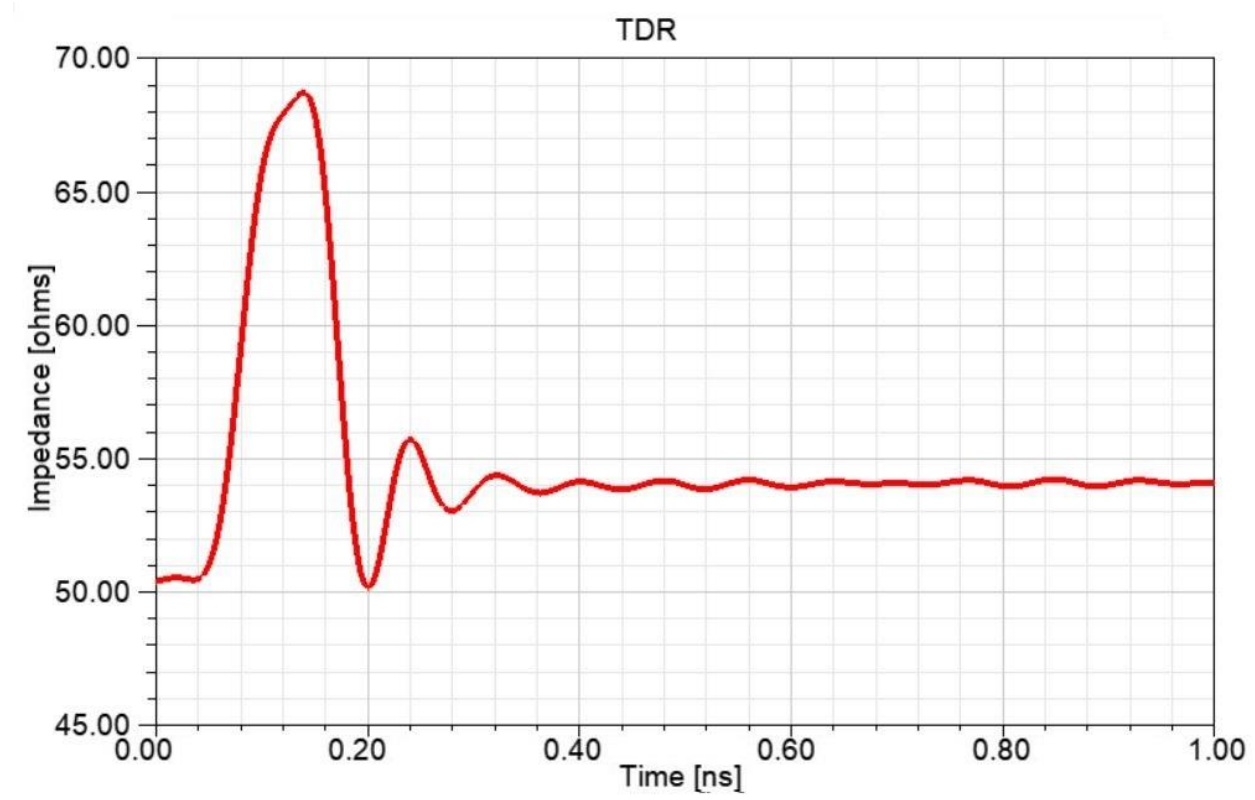

Fig. 20. TDR plot for a high impedance via.

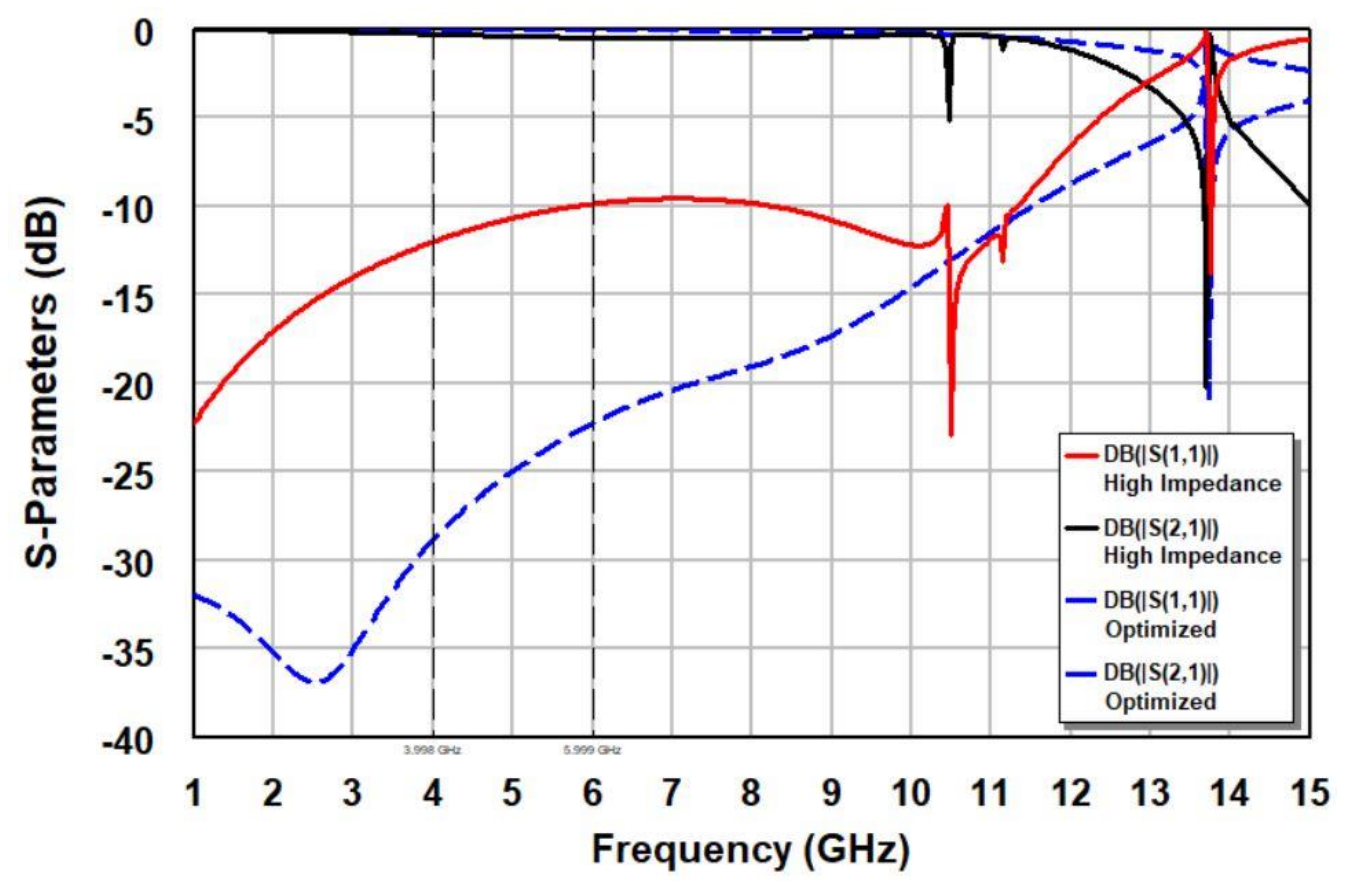

Fig. 21. S-parameters of the high impedance via and the optimized via. 


\subsubsection{Optimized Vias}

For the 1-level, 2-level, and 3-level transitions, arbitrary dimensions were chosen and then fine-tuned using the guidelines in Table 3. These optimized vias are shown in Fig. 22 , and the dimensions are summarized in Table 6 . The guidelines proved to consistent and effective for all variations. Minimizing the impedance mismatch caused by the via yields the TDR plot shown in Fig. 23. Interestingly, all the transitions performed similar to each other and yielded less than $-22 \mathrm{~dB}$ return loss throughout the filter passband as shown in Fig. 24. Fig. 25 shows a significant improvement in return loss.
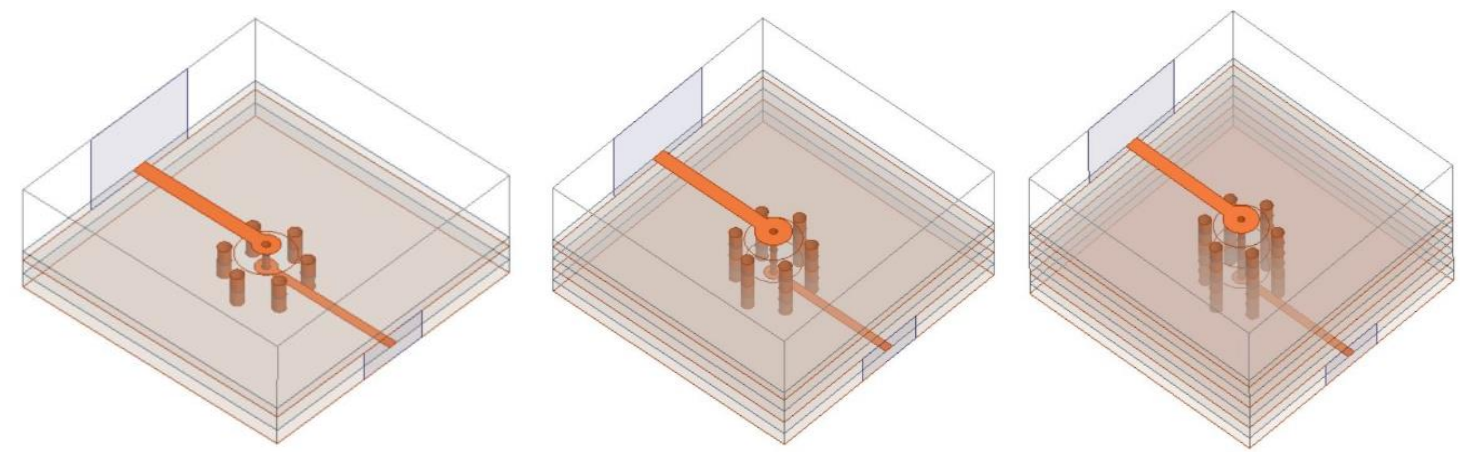

Fig. 22. Optimized via for a 1, 2, and 3 level transition.

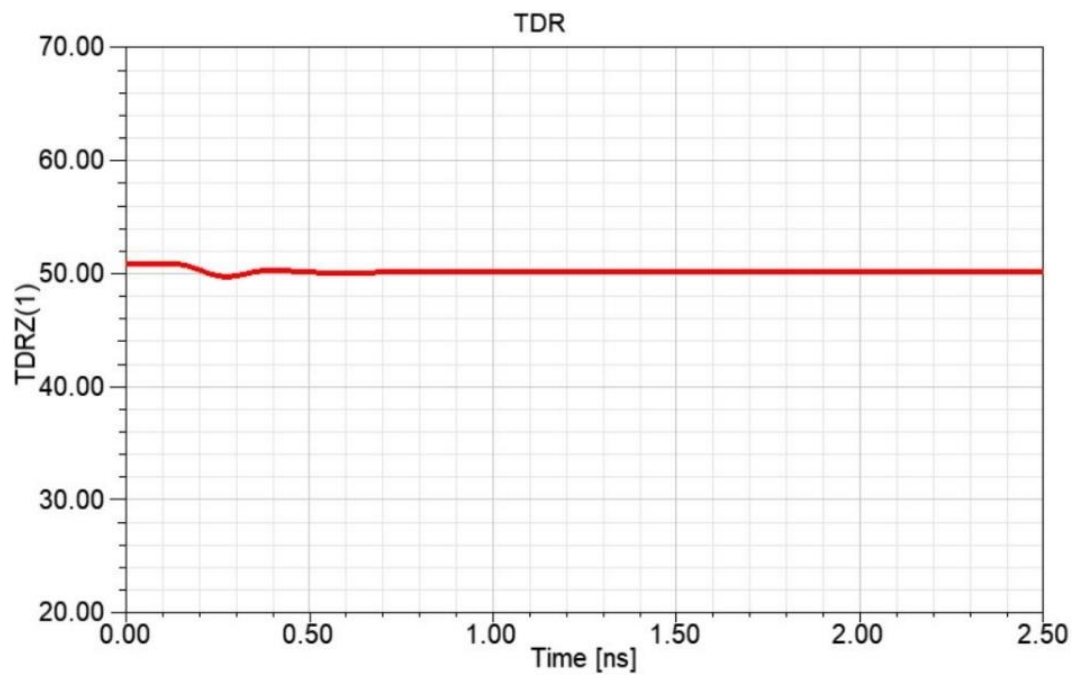

Fig. 23. Optimized via TDR. 
Table 6

Optimized Via Dimensions and Performance

\begin{tabular}{|l|c|c|c|c|c|c|}
\hline & $\begin{array}{c}\text { Passband } \\
\text { Return Loss } \\
(4 \mathrm{GHz}-6 \mathrm{GHz})\end{array}$ & $\begin{array}{c}\text { Size } \\
(\mathrm{mils})\end{array}$ & $\begin{array}{c}\text { Microstrip } \\
\text { Pad Radius } \\
\text { (mils) }\end{array}$ & $\begin{array}{c}\text { Stripline } \\
\text { Pad Radius } \\
\text { (mils) }\end{array}$ & $\begin{array}{c}\text { Via } \\
\text { Radius } \\
\text { (mils) }\end{array}$ & $\begin{array}{c}\text { Antipad } \\
\text { Radius } \\
\text { (mils) }\end{array}$ \\
\hline 1 Level & $<-22 \mathrm{~dB}$ & 168 & 25 & 20 & 7.5 & 54 \\
\hline 2 Level & $<-22 \mathrm{~dB}$ & 168 & 35 & 18 & 7 & 54 \\
\hline 3 Level & $<-22 \mathrm{~dB}$ & 168 & 35 & 18 & 7 & 54 \\
\hline
\end{tabular}

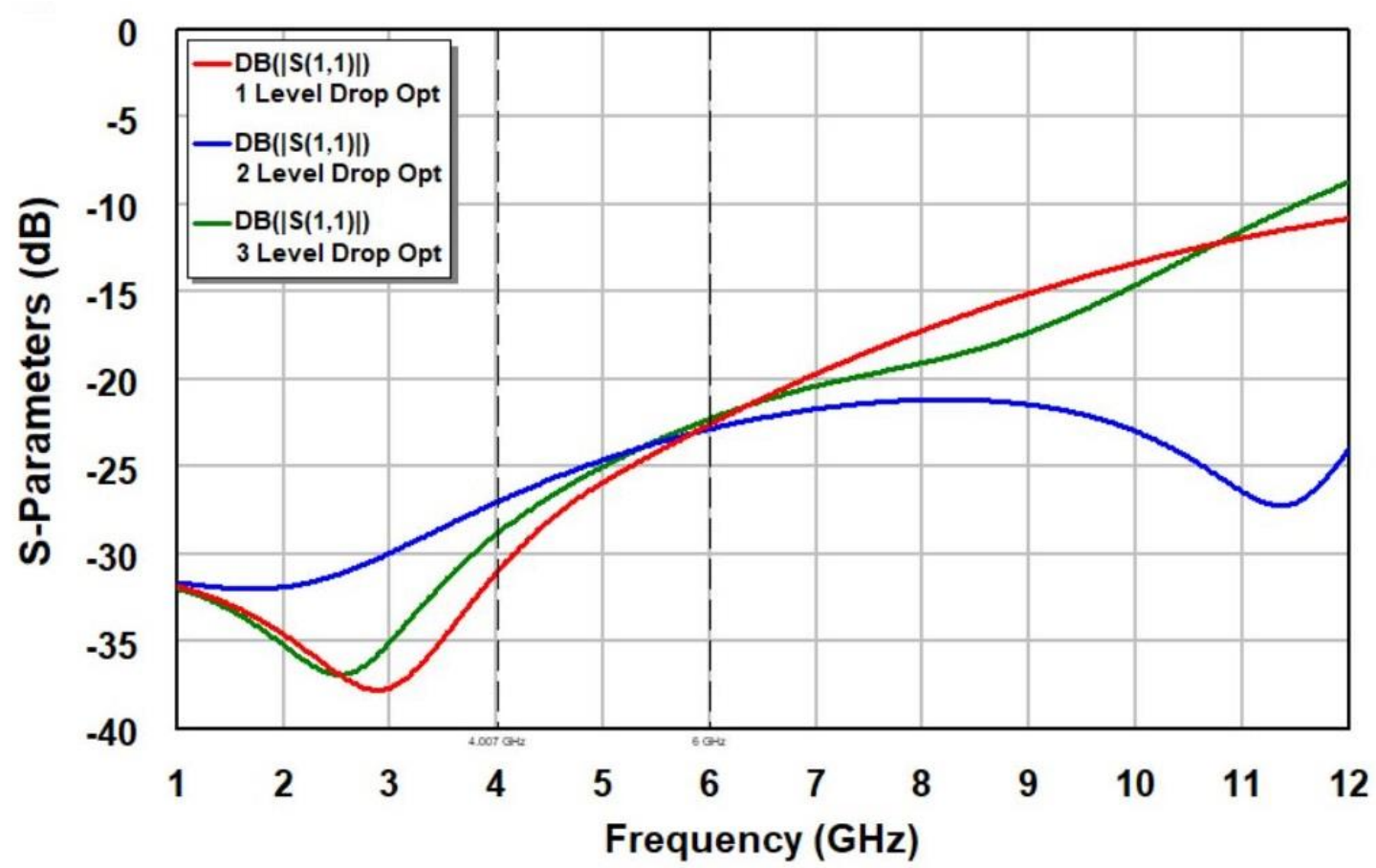

Fig. 24. S-parameters of the optimized 1, 2, and 3 level transitions. 


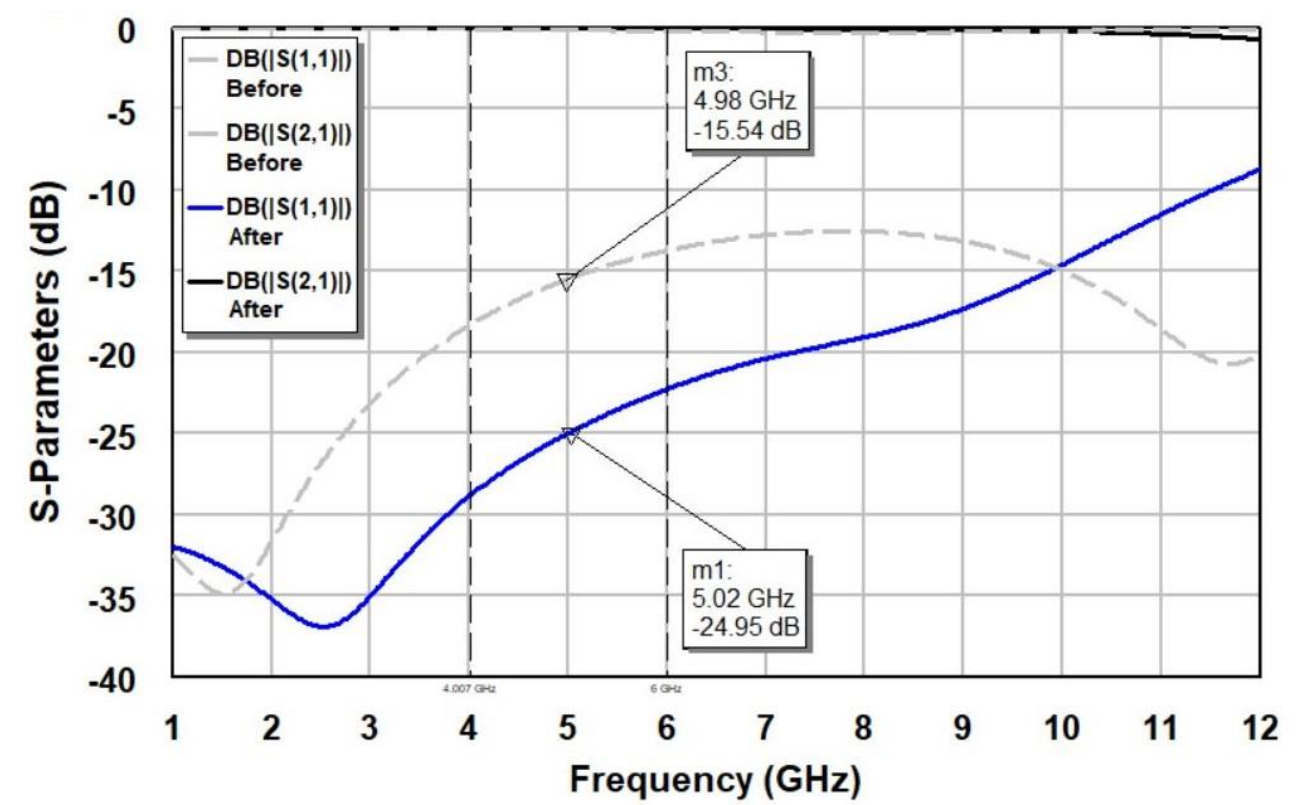

Fig. 25. S-parameters of the 3-level transition before and after optimization.

\subsection{Cascading a Planar Filter}

Since a trace that goes down must come back up, it is useful to also consider the performance of two vias cascaded together. This yields insight on how a passive structure submerged in an inner layer or a crossover of traces may perform. As shown in Fig. 26, cascades of the optimized 1-level, 2-level, and 3-level transition were simulated. From Fig. 27, one can see that all variations maintained a return loss of $-15 \mathrm{~dB}$ up to $8.18 \mathrm{GHz}$. In the filter passband of $4 \mathrm{GHz}$ to $6 \mathrm{GHz}$, a return loss of less than $-17.2 \mathrm{~dB}$ is maintained with all the variations. 

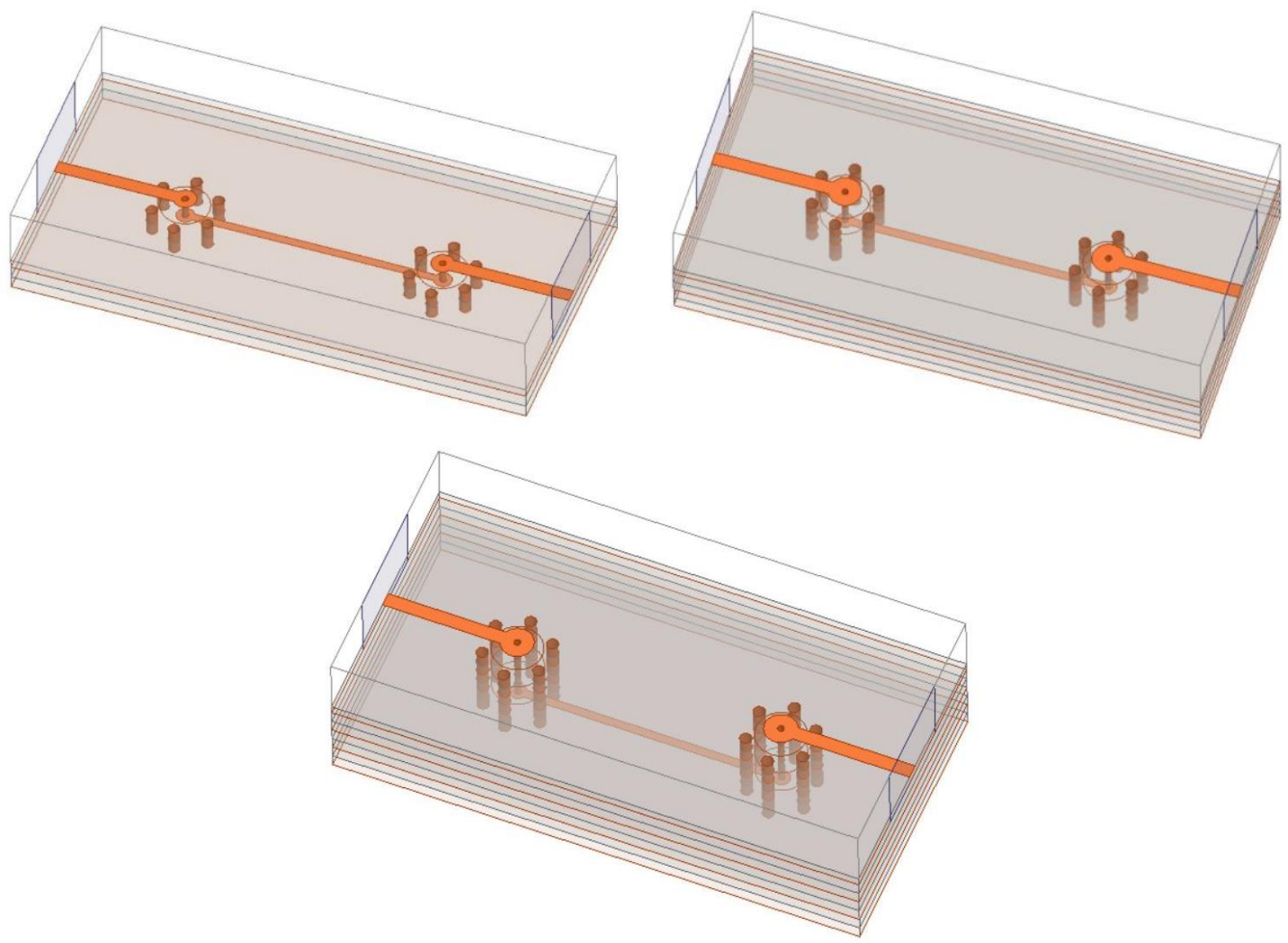

Fig. 26. 3D models of the cascaded 1, 2, and 3 level transitions.

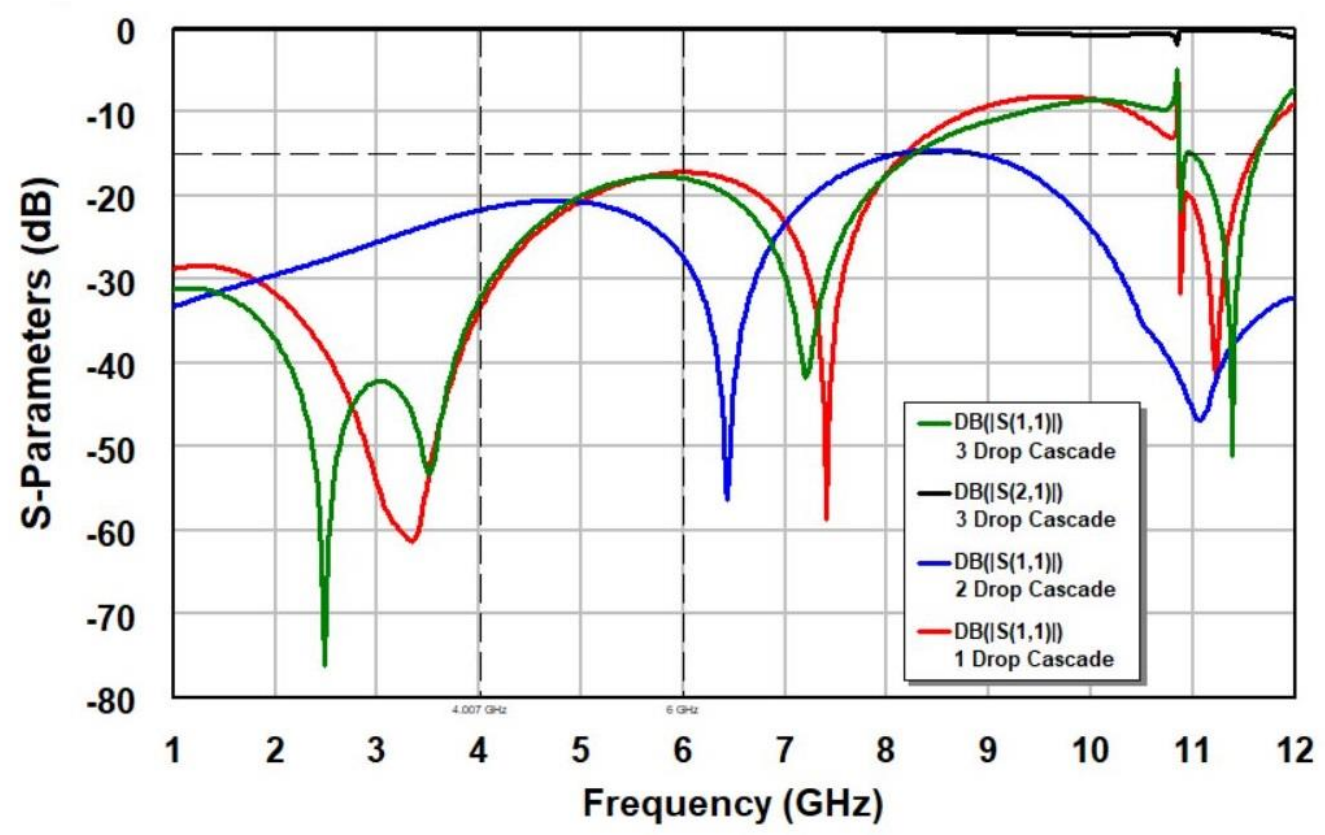

Fig. 27. S-parameters of the cascaded 1, 2, and 3 level transitions. 
To demonstrate the effectiveness of the optimized vertical transitions, a planar 3-pole filter submerged three levels down is simulated. This filter is shown in Fig. 28 and consists of 3 quarter-wave short stubs inductively coupled with thin striplines. The input/output is also inductively coupled with thin striplines. With a dielectric constant of 6.15 , the overall size of the filter is 0.690 " by 0.578 ". Fig. 29 shows the S-parameters of the filter and the performance specifications are summarized in Table 7.

Table 7

Filter Specifications

\begin{tabular}{|c|c|}
\hline Center Frequency & $5 \mathrm{GHz}$ \\
\hline $1 \mathrm{~dB}$ Bandwidth & $4 \mathrm{GHz}$ to $6 \mathrm{GHz}$ \\
\hline Passband Return Loss & $<-20 \mathrm{~dB}$ \\
\hline Size & 0.690 ” by $0.578 ”$ \\
\hline
\end{tabular}

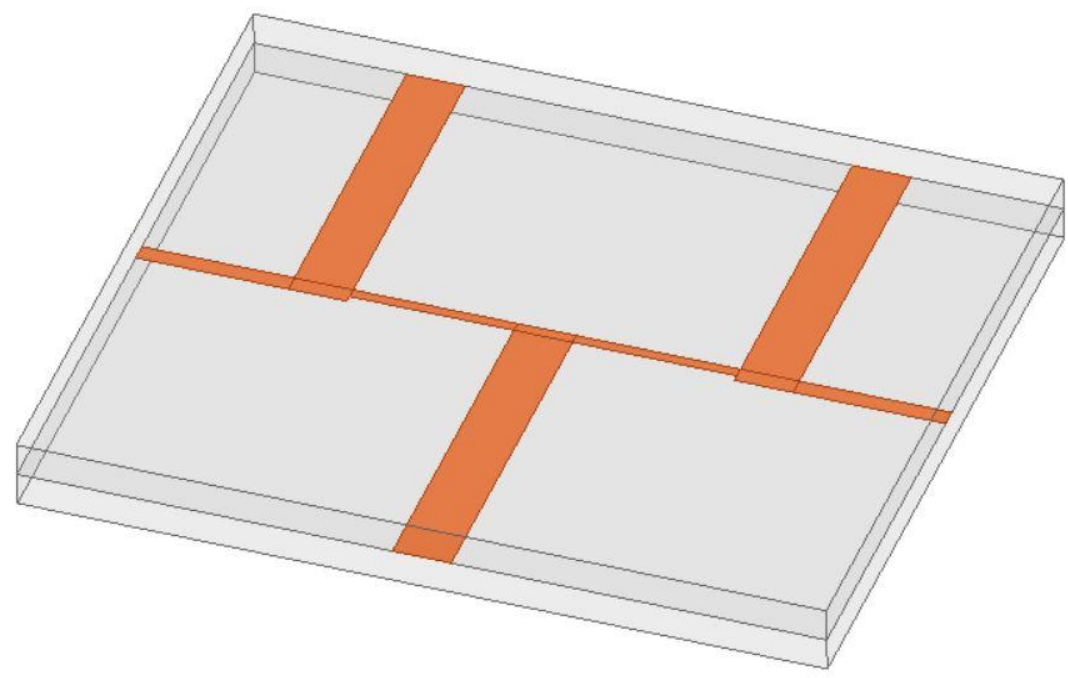

Fig. 28. Planar 3-pole filter. 


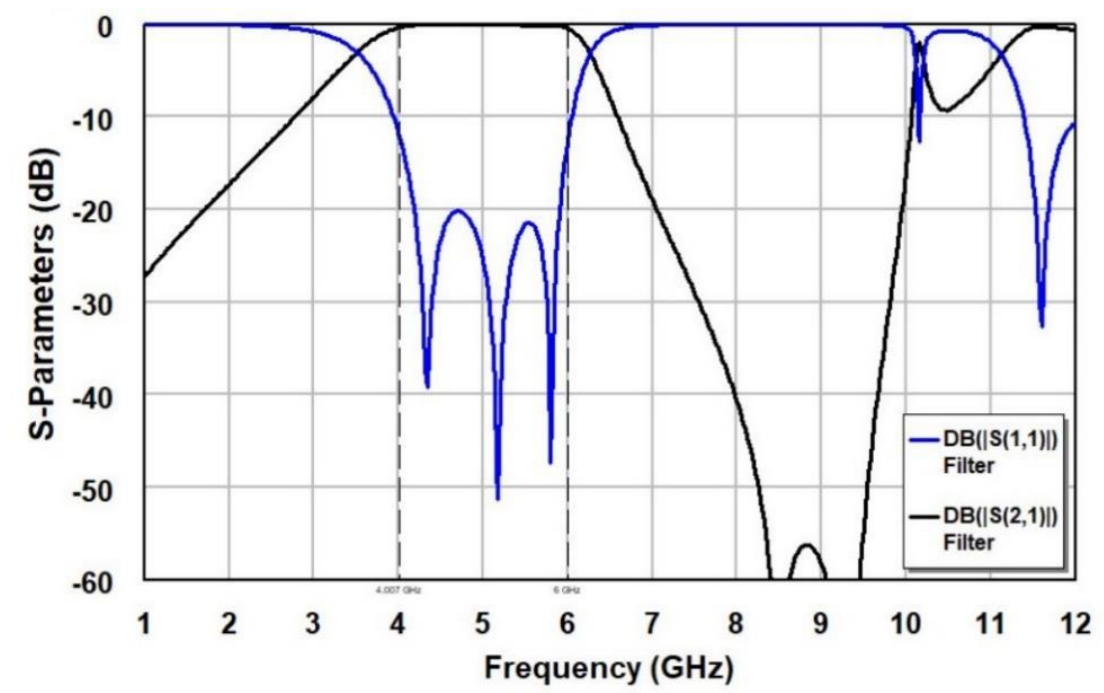

Fig. 29. S-parameters of the planar 3-pole filter before being submerged.

Fig. 30 shows the 3D model of the optimized 3-level drop and the planar 3-pole filter cascaded together. The performance of the filter is mostly retained as shown in Fig. 31. The passband of the filter shifted approximately $100 \mathrm{MHz}$ lower, and the highest passband return loss shifted from $-20.2 \mathrm{~dB}$ to $-14.5 \mathrm{~dB}$. With some minor tuning, the equal ripple was restored, and an overall return loss of $-16.6 \mathrm{~dB}$ was obtained. Fig. 32 shows the S-parameters of the submerged filter after minor tuning.

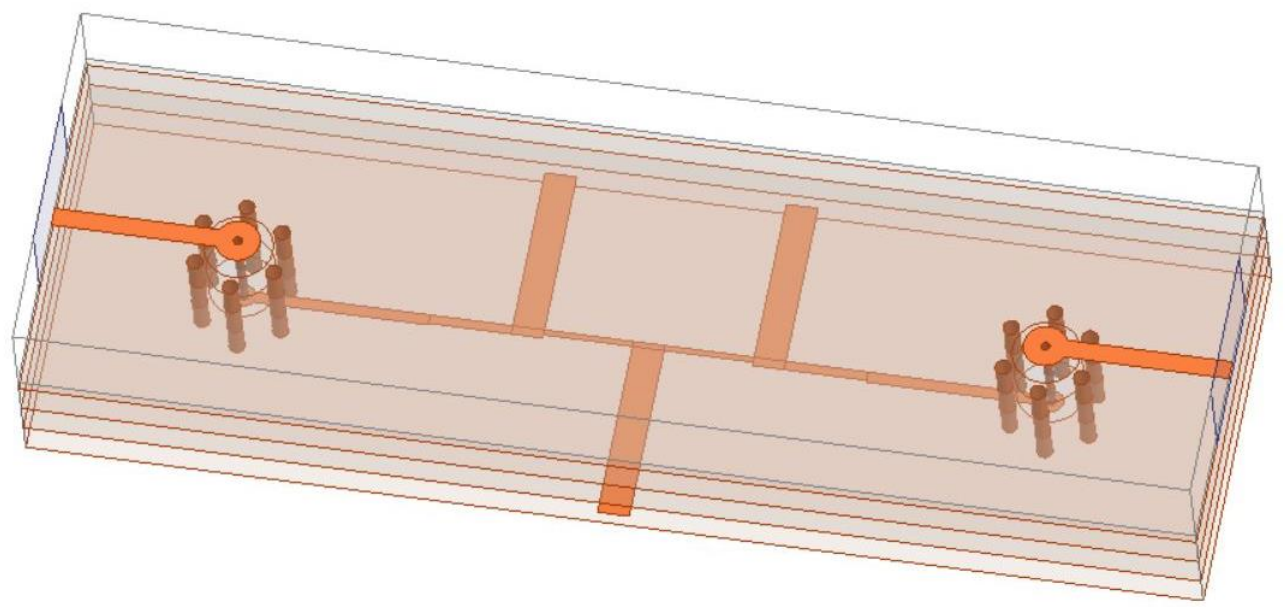

Fig. 30. 3D model of the planar 3-pole filter buried 3 levels in. 


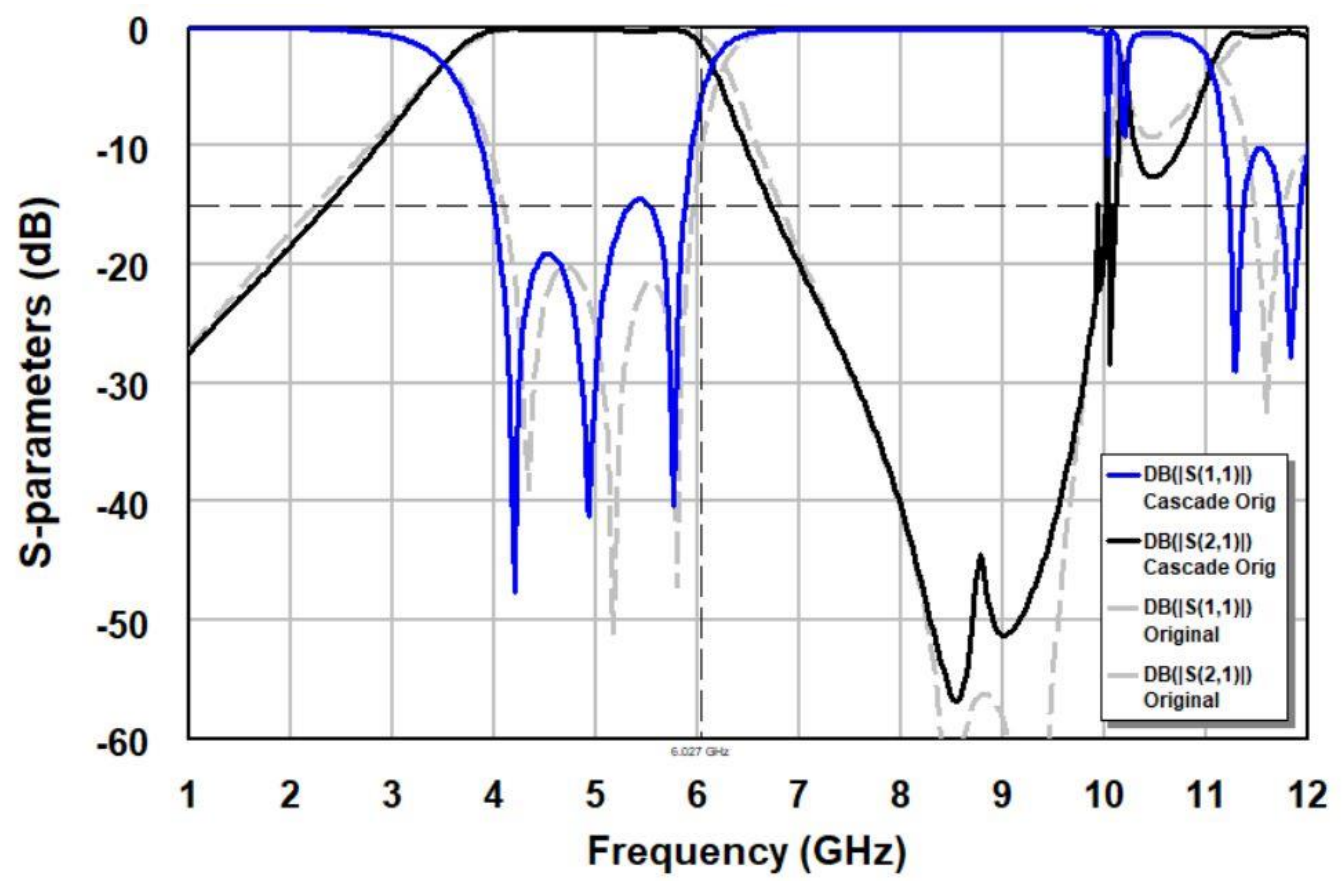

Fig. 31. S-parameters of the submerged planar 3-pole filter before tuning.

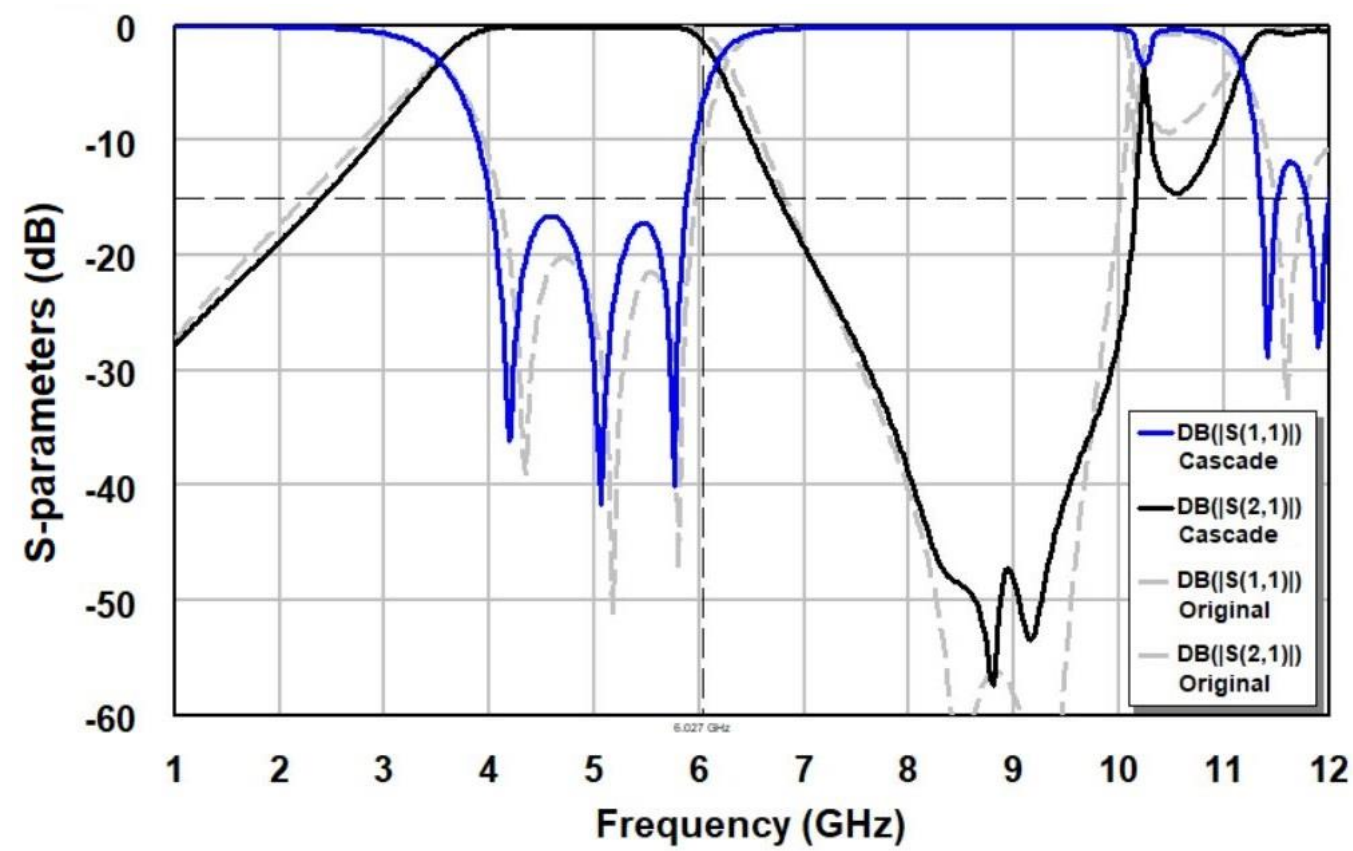

Fig. 32. S-parameters of the submerged planar 3-pole filter after tuning. 


\section{CONCLUSION}

EM simulation showed that for the given multilayer stack-up, 6 stitching vias effectively shielded cavity resonance up to $9.14 \mathrm{GHz}$. Eight vias were effective up to 13.6 GHz. Using more than 8 vias would cause an intersection with the stripline; thus an alternative stitching pattern is needed for higher frequency shielding.

In addition, time-domain reflectometry and the optimization guidelines in Table 3 were effective for minimizing the impedance mismatch caused by vias. Interestingly, an increase in layer transitions did not correlate with performance degradation. The 1,2, and 3 level transitions had similar performance and maintained less than $-20 \mathrm{~dB}$ return loss up to $7 \mathrm{GHz}$. Other publications that tested similar microstrip to stripline transitions report similar performance. Also, the TDR was not useful for isolating each junction of the via and one could not determine precisely which variable to change. Rather, the impedance of the via was clumped together in the TDR.

The cascades of the 1,2, and 3 level transitions-maintained a $-15 \mathrm{~dB}$ return loss up to 8.18 GHz. Although, a return loss of less than $-17.2 \mathrm{~dB}$ was maintained in the filter passband of $4 \mathrm{GHz}$ to $6 \mathrm{GHz}$, the center frequency of the filter shifted approximately $100 \mathrm{MHz}$ lower. Also, the original $-20 \mathrm{~dB}$ equal ripple return loss became distorted. Minor tuning was required to restore the equal ripple, and a return loss of $-16.6 \mathrm{~dB}$ was obtained.

\subsection{Future Work}

This work can be continued by modeling the cavity with grounding vias rather than the airbox. This would determine if stitching vias are able to provide proper ground to 
lower reference planes. In addition, the conductors could be modeled to have finite conductivity to reflect insertion loss.

To extend performance to higher frequencies, one can try using alternative pad shapes such as a taper or bottleneck. In addition, more stitching vias and an alternative stitching pattern could be used to obtain more shielding. Lastly, to isolate each junction of the via in the TDR, one can try using a much faster rise-time. The last step would be to have the design manufactured to compare measurements and simulations. 


\section{Literature Cited}

[1] Anaren, "A Novel Surface-Mount Approach to Crossover Signals," Microwave Journal, 1, February 2000. [Online]. Available:

https://www.microwavejournal.com/articles/2866-a-novel-surface-mountapproach-to-crossover-signals. [Accessed May 3, 2019].

[2] D. Packiaraj, K. J. Vinoy, P. Nagarajarao, M. Ramesh and A. T. Kalghatgi, "Miniaturized Defected Ground High Isolation Crossovers," in IEEE Microwave and Wireless Components Letters, vol. 23, no. 7, pp. 347-349, July 2013.

[3] P. David, Microwave Engineering. Hoboken, NJ: Wiley, 2012.

[4] Q. Tang, Y. Wang and C. Christopoulos, "Simulation and Research of the PCB Via Effects," Third International Conference on Natural Computation (ICNC 2007), Haikou, 2007, pp. 110-114.

[5] Eurocircuits, "Making a PCB - PCB Manufacturing Step-by-Step," Eurocircuits, 29, January 2017. [Online]. Available: https://www.eurocircuits.com/making-apcb-pcb-manufacture-step-by-step/. [Accessed March 20, 2019].

[6] J. Coonrod, "High-Frequency Laminates for Hybrid Multilayer PCBs," in The PCB Design Magazine, pp. 30-32, September 2013. [Online]. Available: https://www.rogerscorp.com/documents/2915/acm/articles/High-FrequencyLaminates-for-Hybrid-Multilayer-PCBs.pdf. [Accessed February 2, 2019].

[7] J. Browne, "Picking Materials for Multilayer PCBs," in Microwaves and RF, 17, February 2011. [Online]. Available: https://www.mwrf.com/materials/pickingmaterials-multilayer-pcbs. [Accessed March 13, 2019].

[8] J. Coonrod, "Navigating Multilayer Microwave PCB Tradeoffs," in Microwaves and $R F$, pp. 107-114, May 2017. [Online]. Available:

https://www.rogerscorp.com/documents/2329/acm/articles/NavigatingMultilayer-Microwave-PCB-Tradeoffs.pdf. [Accessed February 20, 2019].

[9] M. Graham, High-Speed Digital Design, a Handbook of Black Magic. Englewood Cliffs, NJ: Prentice Hall PTR, 1993.

[10] Qiu Xiaofeng, Wu Yushu, Li Shufang, Ying Chenguang and Gao Yougang, "Simulation and Analysis of Via Effects on High-Speed Signal Transmission on 
PCB," 2004 Asia-Pacific Radio Science Conference, 2004. Proceedings., Qingdao, China, 2004, pp. 283-286.

[11] G. Dong, Y. Biao, D. Xidong and L. Yuan, "Research on the Influence of Vias on Signal Transmission in Multilayer PCBs," 2017 13th IEEE International Conference on Electronic Measurement \& Instruments (ICEMI), Yangzhou, 2017, pp. 406-409.

[12] M. Pajovic, J. Yu and D. Milojkovic, "Analysis of Via Capacitance in Arbitrary Multilayer PCBs," in IEEE Transactions on Electromagnetic Compatibility, vol. 49, no. 3, pp. 722-726, Aug. 2007.

[13] I. N. Ndip, W. John and H. Reichl, "RF/Microwave Modeling and Comparison of Buried, Blind and Through Hole Vias," Proceedings of 6th Electronics Packaging Technology Conference (EPTC 2004) (IEEE Cat. No.04EX971), Singapore, 2004, pp. 643-648.

[14] G. Hernandez-Sosa, R. Torres-Torres and A. Sanchez, "Impedance Matching of Traces and Multilayer Via Transitions for On-Package Links," in IEEE Microwave and Wireless Components Letters, vol. 21, no. 11, pp. 595-597, Nov. 2011.

[15] Z. Li, P. Wang, R. Zeng and W. Zhong, "Analysis of Wideband Multilayer LTCC Vertical Via Transition for Millimeter-Wave System-in-Package," 2017 18th International Conference on Electronic Packaging Technology (ICEPT), Harbin, 2017, pp. 1039-1042.

[16] J. Coonrod, "Influence of Through Hole Vias on PCB RF Performance," in IPC Apex, April 2016. [Online]. Available: https://www.rogerscorp.com. [Accessed February 18, 2019]

[17] E. R. Pillai, "Coax Via-A Technique to Reduce Crosstalk and Enhance Impedance Match at Vias in High-Frequency Multilayer Packages Verified by FDTD and MoM Modeling," in IEEE Transactions on Microwave Theory and Techniques, vol. 45, no. 10, pp. 1981-1985, Oct. 1997.

[18] Wusheng Ji, Xuedong Wang and Ying Li, "Simulation of Scattering Parameter of Signal Via with Shielding Vias," Proceedings of 2005 IEEE International Workshop on VLSI Design and Video Technology, 2005., Suzhou, China, 2005, pp. 94-96. 
[19] C. Tsai, Y. Cheng, T. Huang, Y. A. Hsu and R. Wu, "Design of Microstrip-toMicrostrip Via Transition in Multilayered LTCC for Frequencies up to 67GHz," in IEEE Transactions on Components, Packaging and Manufacturing Technology, vol. 1, no. 4, pp. 595-601, April 2011.

[20] DaeHan Kwon et al., "Characterization and Modeling of a New Via Structure in Multilayered Printed Circuit Boards," in IEEE Transactions on Components and Packaging Technologies, vol. 26, no. 2, pp. 483-489, June 2003.

[21] F. P. Casares-Miranda, C. Viereck, C. Camacho-Penalosa and C. Caloz, "Vertical Microstrip Transition for Multilayer Microwave Circuits with Decoupled Passive and Active Layers," in IEEE Microwave and Wireless Components Letters, vol. 16, no. 7, pp. 401-403, July 2006.

[22] M. Liang, Xiaoju Yu, C. Shemelya, E. MacDonald and H. Xin, "3D Printed Multilayer Microstrip Line Structure with Vertical Transition Toward Integrated Systems," 2015 IEEE MTT-S International Microwave Symposium, Phoenix, AZ, 2015, pp. 1-4.

[23] F. Cai, Y. Chang, K. Wang, C. Zhang, B. Wang and J. Papapolymerou, "LowLoss 3D Multilayer Transmission Lines and Interconnects Fabricated by Additive Manufacturing Technologies," in IEEE Transactions on Microwave Theory and Techniques, vol. 64, no. 10, pp. 3208-3216, Oct. 2016. 